\title{
Surgical Treatment of Low-Lying Rectal Cancer: Updates
}

\author{
Cristopher Varela ${ }^{1}$, Nam Kyu Kim ${ }^{2}$ \\ ${ }^{1}$ Coloproctology Unit, Department of General Surgery, Hospital Dr. Domingo Luciani, Caracas, Venezuela; ${ }^{2}$ Department of Surgery, Severance \\ Hospital, Yonsei University College of Medicine, Seoul, Korea
}

Despite innovative advancements, distally located rectal cancer remains a critical disease of challenging management. The crucial location of the tumor predisposes it to a circumferential resection margin (CRM) that tends to involve the anal sphincter complex and surrounding organs, with a high incidence of delayed anastomotic complications and the risk of the pelvic sidewall or rarely inguinal lymph node metastases. In this regard, colorectal surgeons should be aware of other issues beyond total mesorectal excision (TME) performance. For decades, the concept of extralevator abdominoperineal resection to avoid compromised CRM has been introduced. However, the complexity of deep pelvic dissection with poor visualization in low-lying rectal cancer has led to transanal TME. In contrast, neoadjuvant chemoradiotherapy (NCRT) has allowed for the execution of more sphincter-saving procedures without oncologic compromise. Significant tumor regression after NCRT and complete pathologic response also permit applying the watch-and-wait protocol in some cases, now with more solid evidence. This review article will introduce the current surgical treatment options, their indication and technical details, and recent oncologic and functional outcomes. Lastly, the novel characteristics of distal rectal cancer, such as pelvic sidewall and inguinal lymph node metastases, will be discussed along with its tailored and individualized treatment approach.

Keywords: Rectal neoplasms; Proctectomy; Lymph node excision; Surgical procedures

\section{INTRODUCTION}

The most difficult issues present in the surgical management of distal rectal cancer with a concern about oncologic and functional safety are always being considered in this challenging area for colorectal surgeons [1]. The management of distal rectal cancer remains a debate. Low rectal cancer is defined as a tumor located less than $6 \mathrm{~cm}$ from the anal verge. However, other studies arbitrarily describe it as a tumor less than $5 \mathrm{~cm}$ from the anal verge [2, $3]$. In the past, the only method to evaluate the extent of rectal cancer at the distal rectum was a digital rectal exam. However, the implementation of abdominal computed tomography (CT) scan showed clear images of the local spread of distal rectal cancer,

Received: Oct 26, 2021 - Revised: Nov 16, 2021 - Accepted: Nov 17, 2021 Correspondence to: Nam Kyu Kim, M.D., Ph.D., FRCS, FACS, FRSCRS (Hon.) Department of Surgery, Severance Hospital, Yonsei University College of Medicine, 50-1 Yonsei-ro, Seodaemun-gu, Seoul 03722, Korea

Tel: +82-2-2228-2117, Fax: +82-2-313-8289

E-mail: namkyuk@yonsei.ac

ORCID: https://orcid.org/0000-0003-0639-5632

(C) 2021 The Korean Society of Coloproctology

This is an open-access article distributed under the terms of the Creative Commons Attribution NonCommercial License (https://creativecommons.org/licenses/by-nc/4.0) which permits unrestricted noncommercial use, distribution, and reproduction in any medium, provided the original work is properly cited. where the exact relation between the tumor and surrounding organ used to be unclear [4]. The endorectal ultrasonographic evaluation was later available, easy to use, and helpful to assess the depth of invasion [5]. However, its use was limited and operatordependent, and in some cases, not able to evaluate the complete extension of the tumor above the anal sphincter and levator ani complex. Fortunately, imaging modalities have substantially improved local staging and risk stratification [6]. Magnetic resonance imaging (MRI) has allowed a high-resolution visualization of the extent of the tumor, lymph node (LN) spread, and involvement of the neighboring organs. Moreover, extramural vascular invasion and a positive circumferential resection margin (CRM) on MRI have become critical prognostic factors of recurrence and survival [7-9].

Why do patients and colorectal surgeons have serious concerns about the surgical treatment of distal rectal cancer? Based on my point of view, functional and oncologic outcomes sometimes frustrate us, and many complex situation events occur more frequently in low-lying rectal cancer than upper and middle rectal cancer. Compared to upper rectal cancer, oncologic outcomes for distal rectal cancer are associated with a higher rate of local and systemic recurrence, poorer survival and also poor functional outcomes, and inferior quality of life (QoL) in patients with local 
recurrence $[10,11]$. QoL could be highly compromised in a surgical field where many urogenital structures are located, such as the prostate gland, vagina, and trigone of the urinary bladder and anal sphincter complex, including the pelvic floor muscle $[1,12]$. To choose the most optimal surgical option for an individual, we must consider the patient's age, general condition, even job, etc., to ensure good oncological outcomes and good QoL.

The key to determining the extent of surgery is based on the relation between tumors and adjacent organs, whether they are invaded or not [13-15]. The extralevator abdominoperineal excision (ELAPE) concept appeared for more radical resections, modifying the classic Mile's abdominoperineal resection (APR) technique for getting R0 resection [16-18]. From the past, the most challenging part of this deep dissection was poor visualization. However, the high-resolution images by laparoscopy or robotic surgery provide a better anatomical view, allowing more function-preserving concepts and techniques [19].

Surgical treatment for distal rectal cancer is known to be a technically demanding procedure. Techniques are based on the accurate understanding of the anorectal anatomy, and it depends on high-quality surgery in terms of oncologic and functional outcomes. In addition, lymphatic dissemination of distal rectal cancer involves the pelvic sidewall LN and occasionally the inguinal LNs (ILNs), where dissection remains debatable [20]. Based on patient status, a tailored, individualized treatment approach is of utmost importance when considering oncologic and functional outcomes. Neoadjuvant chemoradiotherapy (NCRT) has been performed on distal rectal cancer other than upper rectal cancer [21]. We frequently observe significant tumor regression and complete pathologic response, permitting organ-preserving strategies.

Despite the emergence of cutting-edge advancements, the management of low-lying rectal cancer remains challenging. The higher risk of local recurrence in low rectal cancer and the high incidence of bowel dysfunction such as fecal incontinence has made its treatment a challenging endeavor [22]. In addition, the critical location of low rectal cancer predisposes it to a CRM that tends to involve the anal sphincters and surrounding organs, pelvic and ILN metastasis, and delayed anastomotic issues associated with radiation therapy (RT). As a result, patients are susceptible to uncontrolled pelvic tumors leading to sepsis, bleeding, fecal incontinence, sexual and urinary dysfunction, and pelvic pain.

In this review article, we would like to summarize recent advances in available techniques and highlight the critical and challenging issues colorectal surgeons confront in the surgical management of low-lying rectal cancer.

\section{CASE PRESENTATION}

The first case was a 60-year-old man who presented distal rectal cancer with metastasis of the pelvic sidewall LN. The rectal exam showed an ulcerofungating mass, circumferentially but mainly anterolaterally located at the rectum. Colonoscopy findings and
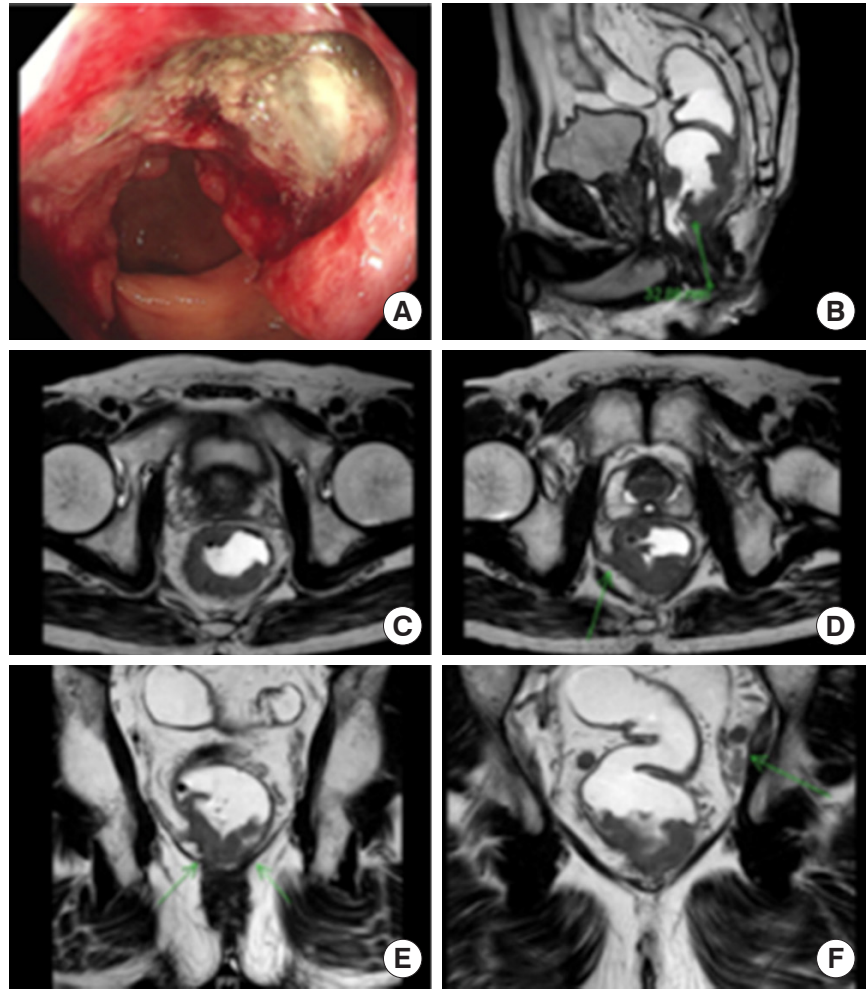

Fig. 1. (A) Colonoscopy finding of low-lying ulcerofungating mass at the anterolateral part of the distal rectum. (B) Rectal magnetic resonance imaging showing mass abutting the prostate gland. (C) Axial imaging of negative circumferential resection margin at prostate level. (D) Tumor extension to the left levator ani muscle. (E) Unilateral tumor involvement of pelvic floor. (F) Enlarged pelvic lymph nodes at the left pelvic sidewall.

rectal MRI (Fig. 1) confirmed a low-lying rectal cancer is mainly anterolaterally located, cT3 or cT4 (anterior and lateral), pelvic sidewall $\mathrm{LN}$ suspicious metastasis $(>8 \mathrm{~mm}$ ) and mesorectal $\mathrm{LN}$ also suspicious metastasis; a coronal view of the rectal MRI showed tumor invasion to the surrounding pelvic floor. We offered standard long-course chemoradiation treatment for downstaging and reducing tumor mass. Follow-up images (Fig. 2) were taken 6 weeks after completion of preoperative long-course NCRT, showing tumor decreased in size and downstaging in the $\mathrm{cT}$ and $\mathrm{cN}$ stage.

We would like to discuss with colleagues several issues in this patient.

1) Anorectal ring level low-lying rectal cancer with risk of +CRM: With a tumor level 3 to $4 \mathrm{~cm}$ above the anal verge, is this patient a candidate for ELAPE? Or a candidate for partial excision of the levator ani muscle (PELM)?

2) Anterior pelvic dissection: Tumor extends laterally to the levator ani and anteriorly to the prostate level. Should we perform a tailored Denovilliers fascia (DVF) excision at the prostate level to 

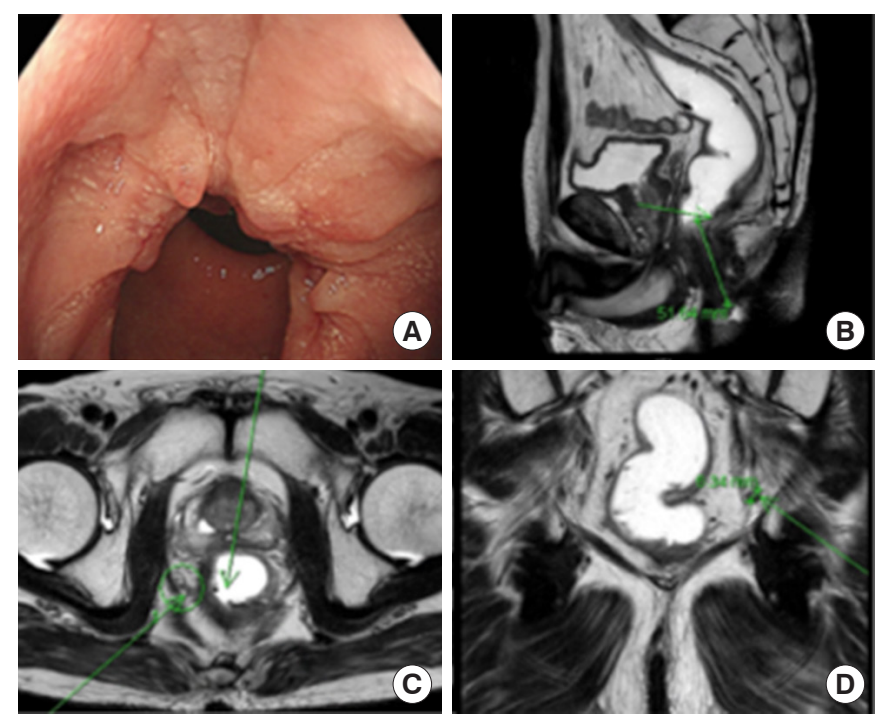

Fig. 2. (A) After neoadjuvant chemoradiotherapy, colonoscopy imaging findings showed marked shrinkage in mass. (B) Rectal magnetic resonance imaging (MRI) showed downstaging for $\mathrm{cT}$ and $\mathrm{cN}$. (C) Axial rectal MRI shows tumor abutting to left levator ani muscle. (D) Coronal rectal MRI shows downsizing of left pelvic sidewall lymph node $(6.3 \mathrm{~mm})$.

secure a negative CRM (Fig. 3 [1])?

3) Is the pelvic sidewall LN metastasis diagnosed on initial MRI an indication for pelvic LN dissection (PLND)? Or should the indication be based on post-NCRT MRI?

In this case, the patient underwent robotic total mesorectal excision (TME) plus ELAPE with anterior DVF excision below the level of the seminal vesicles (tailored excision of DVF) and PLND (Fig. 4).

Let me explain more details about the oncologic extent of surgery and why this treatment option was chosen. A post-irradiated tumor was located from 1 o'clock to 4 o'clock (counterclockwise), involving 4/5 of the rectal lumen $4 \mathrm{~cm}$ above the anal verge and invading the adjacent levator ani muscle. This pelvic floor extension contraindicates ultralow anterior resection or intersphincteric resection (ISR) with coloanal anastomosis (CAA) to get R0 resection, leaving APR, ELAPE, and PELM as treatment options.

To prevent+CRM at the prostate level, anterior pelvic dissection is performed with a tailored resection of DVF. In this case, although preoperative chemoradiotherapy was given, the tumor is still suspicious abutted to the prostate gland, and dissection progresses posteriorly to DVF at the level of the seminal vesicles, and transecting DVF at the prostate level changing dissection to the anterior plane between DVF and the prostate capsule, excising the distal portion of DVF and including it on the specimen. This point is essential to secure an anterior R0 margin and also a way to preserve a neurovascular bundle. Negative CRM at distal rectal cancer is essential, so the DVF excision should be tailored to the tu-
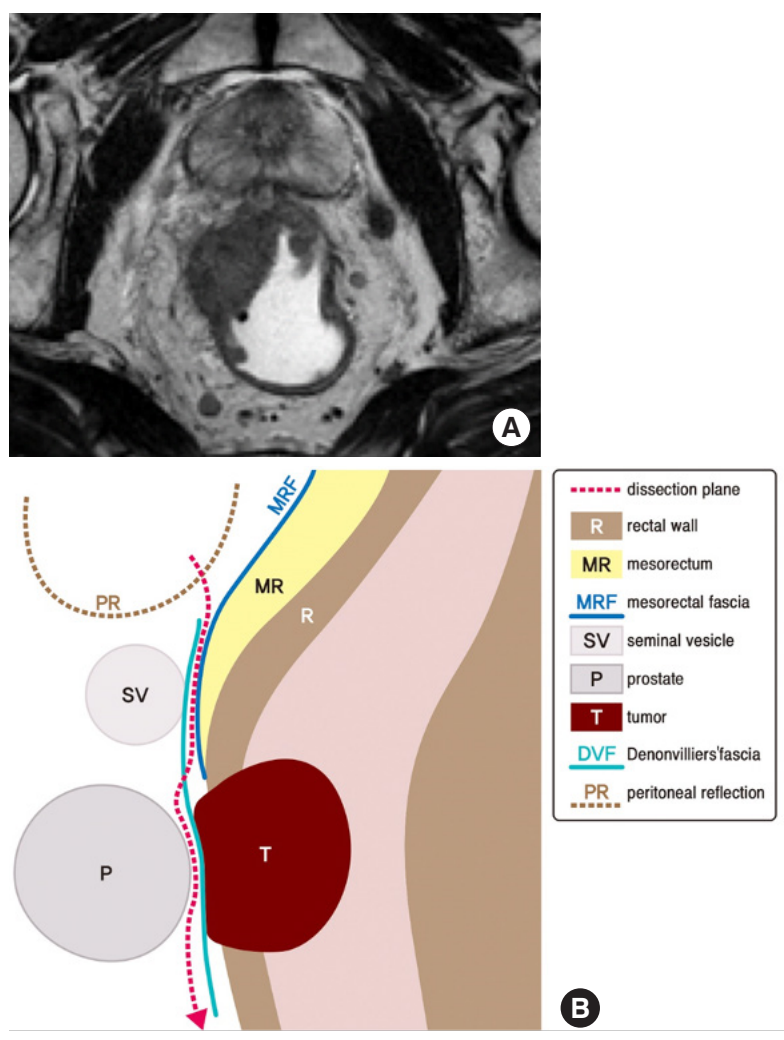

Fig. 3. (A) Tumor abutting the prostate capsule (left) and surgical planes of anterior pelvic dissection with customized excision of Denovilliers fascia at the prostate level. (B) Schematic representation of anterior planes of dissection. Adapted from Kim et al. [1] according to the Creative Commons License.

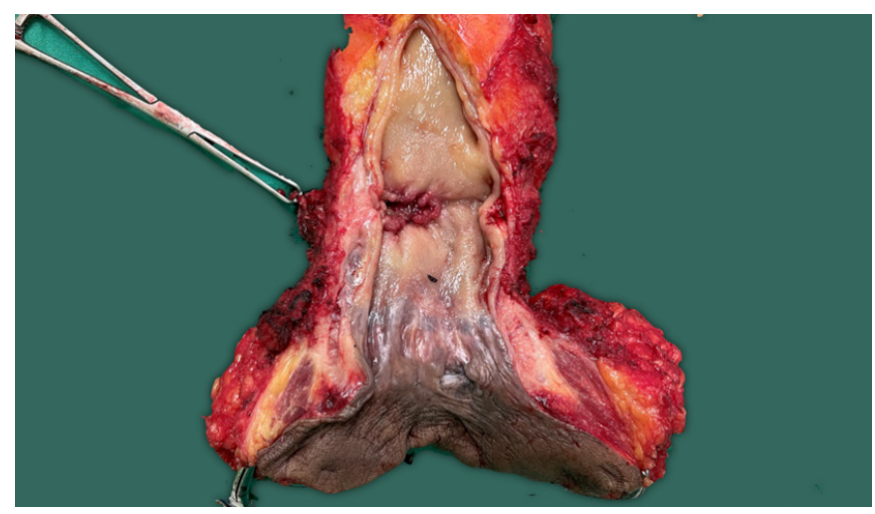

Fig. 4. The tumor center is located anterolaterally at the prostate gland, extending to the right levator ani muscle.

mor level and depth of invasion as a further step in CRM clearing. Initial pre-NCRT measurement of enlarged bilateral pelvic LN by MRI was $>8 \mathrm{~mm}$ in size, indicating bilateral PLND. The final pathologic report showed bilateral pelvic LN were positive for cancer and postoperative, and CT showed no residual metastatic 

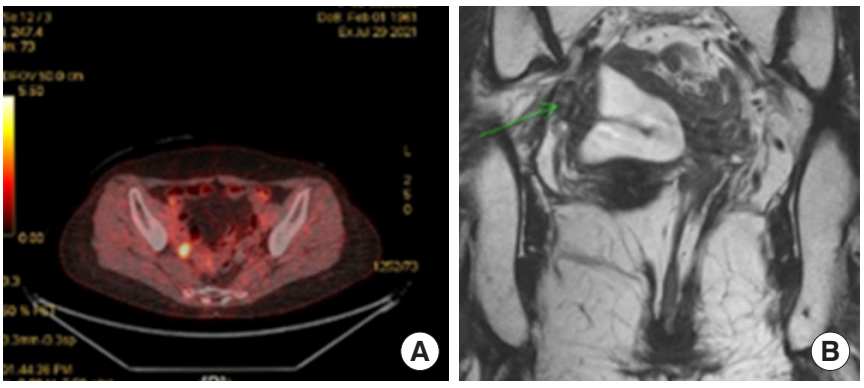

Fig. 5. (A) Positron emission tomography showed fluorodeoxyglucose high uptake at the right pelvic sidewall. (B) Rectal magnetic resonance imaging also showed a metastatic pelvic lymph node at a right pelvic wall.

LN at both pelvic sidewalls in this case. Proper bilateral PLND prevents local recurrence and improves survival outcomes. Indications and debates about PLND after NCRT in rectal cancer will be discussed later.

This low-lying rectal cancer invaded the ipsilateral levator ani muscle extensively, making ELAPE another non-sphincter preserving surgical treatment option for a complete excision of levator ani avoiding+CRM.

The second case is a 60-year-old lady who presented a pelvic LN metastasis as local recurrence. She received a long-course NCRT for rectal cancer between March and April 2018 and underwent robotic intersphinteric resection (ISR) and CAA with right PLND in July 2018. At that time, pathology revealed ypT3N1M0 and received an adjuvant FOLFOX chemotherapy. In August 2021, we diagnosed a local recurrence at the pelvic cavity during follow-up. In Figs. 5 and 6, we can observe a pelvic wall LN recurrence evident in positron emission tomography-CT with fluorodeoxyglucose hot uptake at right pelvic LN, where previously PLND was performed at the time of first surgery. Reviewing the initial rectal MRI (March 2018), an enlarged metastatic pelvis sidewall LN is visible on the right side (Fig. 6A, B). Postoperative follow-up rectal MRI showed the LN remaining on the right pelvic sidewall, evidencing a technical failure of PLND. The technical proficiency of PLND is essential, and that is, in this case, the origin of current pelvic local recurrence. Even if the patient received NCRT, if the PLND was not done in the presence of enlarged LN or performed incompletely, a local recurrence will have a high risk of later appearing. We will discuss the indications and technical proficiency tips for PLND.

\section{SURGICAL MANAGEMENT}

\section{Surgical planning}

Surgical strategies for rectal cancer should aim not only to a curative resection but also to preserve pelvic autonomic plexus and the possibility of anal sphincter preservation with adequate voiding function $[3,23]$.
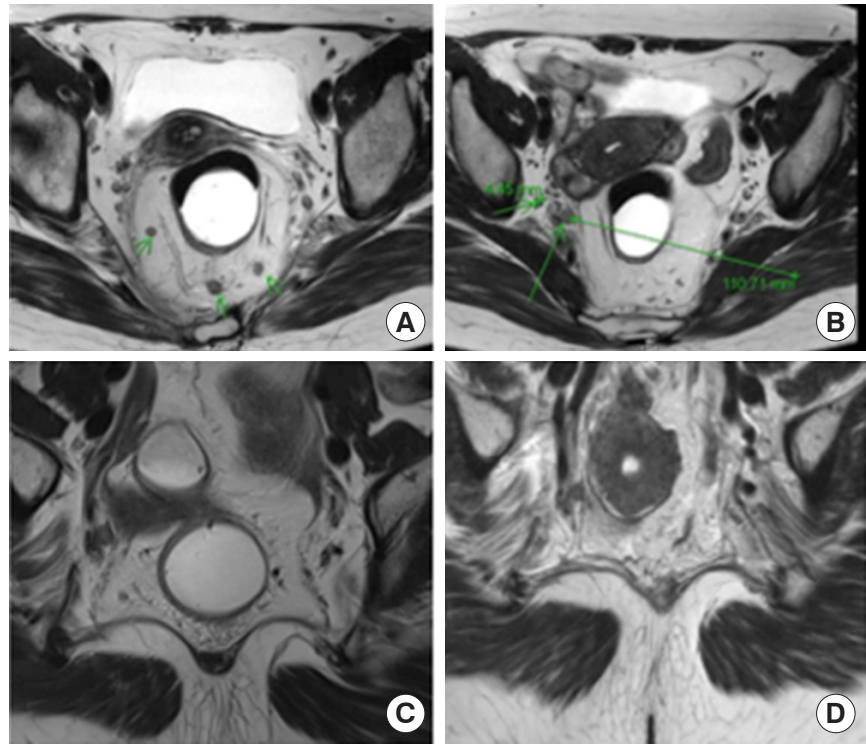

Fig. 6. Rectal magnetic resonance imaging (MRI) showed mesorectal metastatic lymph node (LN) (A) and right metastatic $\mathrm{LN}$ at the pelvic wall (B). After chemoradiotherapy, rectal MRI showed a decrease in the right internal iliac $\mathrm{LN}$ in size; from 7.3 to $4.9 \mathrm{~mm}$, from $4.5 \mathrm{~mm}$ to $4.2 \mathrm{~mm}$ respectively, still metastatic $\mathrm{LN}$ (C). After robotic partial excision of levator ani muscle and right pelvic LN dissection, a remaining right pelvic LN was present in the follow-up rectal MRI 3 months after surgery (D).

TME is a procedure that resects all the mesorectum just above the anal canal, containing the draining LNs and blood vessels surrounding rectal cancer [23]. A tumor-specific mesorectal excision has also been described for partially resecting the mesorectum according to the tumor's location $[24,25]$.

The surgical technique can be summarized in a complete mobilization of the proximal sigmoid colon and descending colon up to the splenic flexure with central vascular ligation inferior mesenteric artery (IMA, high or low ligation) with LN dissection. The mesorectal dissection should be performed between the visceral and parietal fasciae of the pelvis in an avascular plane with identification and preservation of significant structures; common iliac vessels and ureters bilaterally and the origin of the hypogastric nerves near the root of IMA [1].

A negative CRM represents a significant prognostic impact on local recurrence rates, distant metastasis, and survival to achieve a curative resection [26]. A positive CRM increases the rates of local recurrence, metastasis, and death to $40 \%$ exponentially compared with $12 \%$ of negative margin of $\geq 1 \mathrm{~mm}$. There was an exponential increase in local recurrence rates, metastasis, and death with decreasing circumferential margin [26-28].

Sharp dissection of the mesorectum from the surrounding structures must be done to achieve a negative CRM [28]. The DVF has been a classical surgical landmark of anterior dissection, with recent discernments of its contribution to CRM margin in 


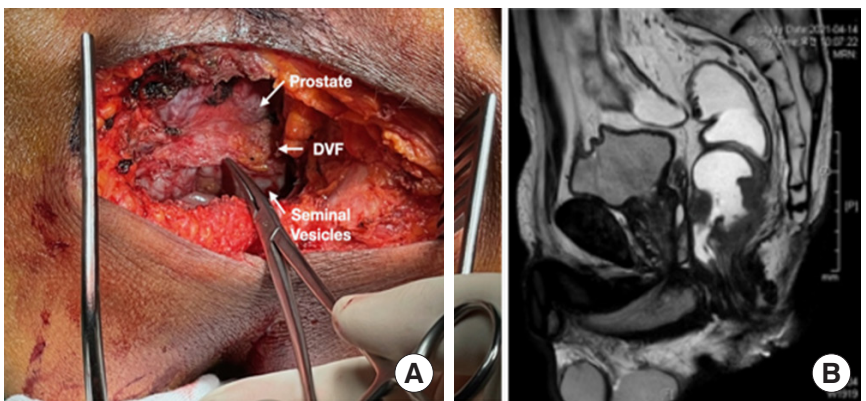

Fig. 7. (A) After specimen resection, the distal portion of the seminal vesicles is observed covered by Denovilliers fascia (DVF), but the prostate capsule is exposed after DVF partial excision at the prostate level. (B) Sagittal rectal magnetic resonance imaging showed the level of the tumor.

anteriorly located tumors [29]. The DVF can be identified during the anterior dissection of the mesorectum as a shiny white layer posterior to the seminal vesicles (Fig. 7), being less evident in female where it is part of the rectovaginal septum [1]. A classification of the available anterior dissection planes of DVF has been proposed comprising 3 anatomical layers in relation with the rectum and the embryological planes of dissection: (1) close rectal plane or perimuscular, a plane within the mesorectum and behind the mesorectal fascia; (2) mesorectal plane, outside the fascia propia of the rectum and posterior to the DVF; and (3) extramesorectal plane, an anterior plane that permits the resection of DVF exposing the prostate and seminal vesicles [29]. Based on this, a customized excision of the DVF according to the tumor level and clinical T stage is believed to have both oncologic and functional significance where partial or complete excision of the DVF can be done dissecting beyond the seminal vesicles and posterior to the prostate [3].

Several meta-analyses have addressed the issue of ligating the IMA at its origin from the aorta (high ligation) or distal to the origin of the left colic artery (low ligation) with concerns in a possible incidence of anastomotic leakage (AL) or different oncological outcomes regarding the total number of LNs harvested finding no difference between high and low ligation in postoperative outcomes $[30,31]$.

Sphincter-preserving surgery (SPS) can be indicated when resection with negative CRM and distal resection margins (DRM) can be achieved, and an adequate anal function can be maintained [32,33]. According to the classification described by Rullier et al. [28], SPS can be adapted to the tumor relation to the sphincter complex to achieve a free distal margin of at least $1 \mathrm{~cm}$; for tumors above the anal sphincter complex, the internal sphincter is preserved, and anal mucosectomy is performed above the dentate line (DL). For juxtaanal and intraanal tumors, the internal sphincter is removed partially or totally, respectively, with ISR, including the overlying anal mucosa [28].

Currently, sphincter preservation paradigms are changing with

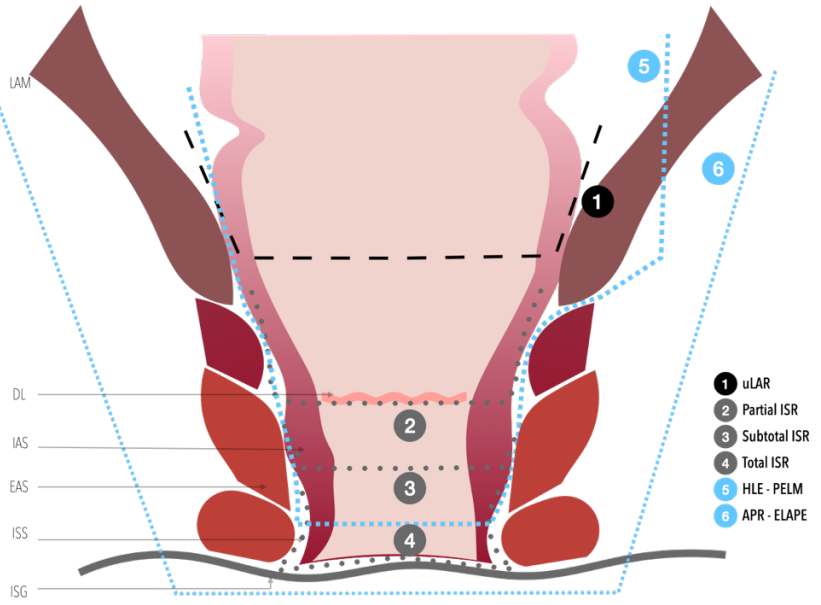

Fig. 8. Surgical strategies for rectal resection. LAM, levator ani muscle; DL, dentate line; IAS, internal anal sphincter; EAS, external anal sphincter; ISS, intersphincteric space; ISG, intersphincteric groove; uLAR, ultralow rectal resection; ISR, intersphincteric resection; HLE, hemilevator excision; PELM, partial excision of LAM; APR, abdominoperineal resection; ELAPE, extralevator abdominoperineal excision.

the positive outcomes of NCRT in ultralow rectal cancer as demonstrated by the GRECCAR 1 prospective trial, where the surgical decision could be safely changed from an initial APR to an SPS after evaluating tumor response to NCRT with no oncologic outcome differences in a 10-year long-term follow-up. Recently, a novel technique has been introduced for SPS for rectal cancer with partial involvement of the unilateral levator ani muscle named PELM. This anatomic condition would have been treated with APR in previous decades [34] (Fig. 8).

\section{Current approaches}

The multimodality approaches for the management of rectal cancer and the operative techniques improvements of minimally invasive procedures, including laparoscopic and robotic TME, have improved the rates of local recurrence and survival after proctectomy [1]. Nevertheless, the standardized surgical steps remain in the different approaches to guarantee optimal oncologic outcomes.

Transanal local excision is indicated for noncircumferential villous adenomas and well to moderately differentiated cTis and cT1 carcinomas [4] without evidence of LN invasion, smaller than 3 $\mathrm{cm}$ in diameter, located between 8 to $10 \mathrm{~cm}$ from the anal margin and occupying less than $1 / 3$ of the circumference of the rectum lumen, that can be accessed transanally for complete transanal excision [35], either by conventional techniques or by transanal endoscopic microsurgery (TEM) or transanal minimally invasive surgery (TAMIS), which is considered the standard procedure for this type of resection with 10 -year overall survival rates of greater than $75 \%$ disease-free survival $[22,36-40]$.

Recent multicenter randomized clinical trials evaluated the on- 
cological outcomes of laparoscopic rectal cancer surgery. The MRC-CLASSIC, COLOR II, and COREAN trials found no differences in local recurrence or disease-free survival rates between laparoscopic and open surgery [10,41, 42]. Although the noninferiority of laparoscopic surgery, concerning open surgery for rectal cancer, was not established on statistical analysis in the ACOSOG Z6051 and ALaCaRT trials [43, 44], the 10-year followup of the COREAN trial confirms the long-term oncological safety of laparoscopic surgery in patients with rectal cancer treated with preoperative chemoradiotherapy [45].

Furthermore, the superiority of robotic surgery over laparoscopic surgery was not proven in the ROLARR trial [19]. As shown in previous studies, advanced surgical techniques could not ensure better oncological outcomes, but they can allow safe performance of deep pelvic dissections [1, 46, 47]. Although the anorectal function has been proved to recover better 1 year after robotic TME when compared to laparoscopic, open, or transanal approach, probably due to better visualization and preservation of pelvic neurovascular structures [48].

\section{Transanal total mesorectal excision Introduction}

Oncological outcomes, particularly long-term local disease control in low-lying rectal cancer, depend on obtaining a high-quality TME specimen with an intact mesorectum with adequate CRM $[49,50]$. Some particular conditions can increase the complexity of transabdominal TME in certain cases; per example, visualization can be complex in an obese patient with a bulky tumor and a narrow pelvis, also pelvic bone parameters such as sacral depth or intertuberous diameter [11], and the incline angle of pelvic floor muscle may provide an anatomical parameter that predicts TME difficulty [51] leaving limited space for adequate pelvic dissection. Furthermore, having an anteriorly located distal tumor can also complicate the situation for these patients by predisposing them to a higher risk of CRM involvement $[52,53]$. The difficult introduction of instruments with a limited range of movement in the pelvis is an obstacle in ensuring a secure distal margin, sometimes requiring multiple stapler firings for resection that can increase the risk of AL [54].

In response to these challenges, transanal TME (TaTME) was developed to provide a more direct approach to the most complicated phases of dissection in the distal rectum. TaTME merges 3 established rectal surgery techniques: TME, transanal transabdominal ISR, and TAMIS [55]. The primary advantage of this technique is that the surgeon can directly visualize and define the DRM of the tumor and enter the mesorectal dissection plane at its most caudal aspect. Direct visualization allows safe dissection around the critical structures that envelop the narrow pelvis, including the vagina, prostate, and pelvic neurovascular structures. Pneumoinflation of the TME plane provides a significant amount of tissue retraction, further facilitating the rectal dissection and mobilization $[55,56]$. TaTME provides short and long-term mor- bidity, functional and oncologic outcomes similar to laparoscopic TME (LapTME) $[22,55]$. Recent studies showed that this "bottom-to-up" approach could reduce a positive rate of CRM, get an adequate DRM, and get a better complete TME specimen [22].

\section{Patient selection}

TaTME approach is optimally designed for men, patients with the narrow and deep pelvis, visceral obesity or body mass index (BMI) of $>30 \mathrm{~kg} / \mathrm{m}^{2}$, prostatic hypertrophy, tumor diameter of $>4 \mathrm{~cm}$, distorted tissue planes such as irradiated fields, challenging to palpate tumors, and failure to progress from a traditional open or laparoscopic operative approach [55].

A consensus about the indications of TaTME was published after the second International Transanal Total Mesorectal Excision Conference [57]. Experts recommended TaTME in patients with the following characteristics: male, narrow and deep pelvis, obese, tumor less than $4 \mathrm{~cm}$ from anal verge, prostate enlargement, and distorted planes caused by irradiation. However, more objective parameters are necessary to predict transabdominal TME difficulty and develop a better objective parameter that can give clear indications for TaTME for middle and distal rectal cancer.

\section{Surgical technique}

TaTME is performed through an access sleeve into the anal canal; a purse-string is used to close the rectal lumen either via an open method with a standard needle driver or a laparoscopic through the access channel, utilizing luminal insufflation [56, 57]. With a closed lumen, full-thickness, circumferential division of the rectum is performed using electrocautery $1 \mathrm{~cm}$ distal to the pursestring stitch entering the TME dissection plane and progressed cranially until the abdominal cavity is entered. Via laparoscopy, an abdominal team provides tissue retraction and exposure besides completing the colon ligation and mobilization. Extraction of the specimen and anastomosis can be performed according to the surgeon's choice, the patient's anatomy, and tumor/specimen bulk $[56,57]$.

\section{Postoperative complications}

Recent studies from the International TaTME registry showed issues about anastomotic failure and urethral injury had been reported. The rates of anastomotic failures, such as leaks, pelvic abscesses, anastomotic fistulas, and strictures, as well as chronic sinuses, have reported an overall 30-day anastomotic leak rate of $7.8 \%$ and a delayed leak rate of $2.0 \%$, with an overall anastomotic failure rate of $15.7 \%$ to $20 \%$ [58, 59]. Several stapled and handsewn techniques have been reported to perform an anastomosis after TaTME. Still, this leak rate and overall morbidity remain within an acceptable range compared to laparoscopic or robotic transabdominal TME [58].

Incorrect anterior dissection plane near the prostate urethra results in urethral injury. Sylla et al. [60] reported urethral and other urologic injuries during TaTME. The low rectal cancer develop- 
ment program International TaTME registry $[50,61]$ reported a $0.8 \%$ incidence, 34 urethral, 2 ureteral, and 3 bladder injuries were reported. The urethral repair complication rate was $26 \%$, with $9 \%$ of failed urethral repair requiring permanent urinary diversion; in patients with successful repair, $18 \%$ reported persistent urinary dysfunction.

Structured training such as case observation, video-based didactics, and proctoring of the first few cases are needed to improve operative outcomes. Persiani et al. [62] described the learning curve for TaTME, finding that anastomotic complications started to decrease after 27 cases and the major complication rates decreased after the 54th case and the mean operative time decreased after the 71 st case.

\section{Oncologic outcomes}

A recent prospective clinical trial compared the outcomes of TaTME vs. LapTME [50] in 18 patients operated by TaTME vs. 20 patients by LapTME. Results found a significantly higher BMI in the TaTME group than in the LapTME group $(\mathrm{P}=0.03)$. TaTME was associated with more transanal specimen extraction $(55.5 \%$ vs. $20 \%, \mathrm{P}=0.06$ ). No significant differences were detected in CRM, DRM, peri- or postoperative complications, or conversion rates with more reported Clavien-Dindo grade III complications in the TaTME group $(\mathrm{P}=0.29)$. In the Netherlands, a prospective clinical trial [49] compared TaTME vs. LapTME with better oncologic outcomes in the TaTME group, reporting a positive CRM he resection margin in $12.2 \%$ of LapTME, vs. $4.9 \%$ of TaTME cases $(\mathrm{P}=0.432)$. The TME specimen quality was complete in $84.0 \%$ of the laparoscopic cases and $92.7 \%$ of TaTME cases $(\mathrm{P}=0.266)$.

On the other hand, a national decision statement was published by the Norwegian Colorectal Cancer Group in 2018 [63] after evidencing an unexpectedly high rate of early recurrences after TaTME (11.6\% estimated local recurrence rate at 2.4 years) with rare multifocal growth on the pelvic sidewalls and cavity, theorizing the possibility of tumor cell spread by the airflow during dissection after rectal transection and possible looseness of the purse-string suture or technical problems like dissection in a wrong plane. In the same cohort, anastomotic leak rates after TaTME were higher than national rates for TME (8.4\% of patients in the TaTME cohort compared with 4.5\% of TME patients) [64]. These adverse events could be related to a progressive learning curve of the procedure, given the technical difficulties related to a bottom-to-up approach of the mesorectum; nevertheless, there was no difference in local recurrence among Norwegian highvolume and low-volume TaTME centers, raising the concern of oncologic or technical procedure-related complications that are still needed to define $[34,63]$. Currently, results are being expected from the COLOR III trial, an ongoing international, multicenter, superiority, randomized trial designed to compare TaTME and conventional LapTME as the surgical treatment of mid and low rectal carcinoma [65].

\section{Functional outcomes}

The potential postoperative urinary dysfunction and poor bowel function after TaTME could affect the QoL beyond the oncologic benefits and should be discussed with the patient in the preoperative setting [66]. In this matter, 2 recent meta-analyses have compared functional anorectal and urologic outcomes between TaTME and LapTME [67, 68]; both studies did not find significant differences regarding urologic outcomes (International Prostate Syndrome Scores of 5.5 to 8.0 in the TaTME group, and 3.5 to 10.1 in the LapTME group) and anorectal functional outcomes were similar in both groups with concerning low anterior resection syndrome (LARS) scores but significantly higher in the TaTME group. In the long term, both techniques have similar oncological and functional outcomes and QoL. High LARS scores might be related to a low coloanal or colorectal anastomosis, prolonged dilatation of the external anal sphincter, and partial or total resection of the internal anal sphincter (IAS) during the procedure.

\section{Expert comments}

To overcome the learning curve and avoid surgical complications with good oncologic outcomes, adequate step-by-step training and expert proctoring are needed. This should be kept in mind to safely implement a technique proven to help the colorectal surgeon get successful outcomes in ultralow rectal cancers or complex pelvis. The favorable short-term oncologic outcomes reported for TaTME are associated with better complete TME specimen, a more extended DRM, and less positive CRM. Long-term follow-up and ongoing randomized controlled trials data awaited regarding functional outcomes, local recurrence, and survival.

Especially COLOR III trial, which aims to compare TaTME with LapTME, exclusion criteria for TaTME were cT3 with margin of $<1 \mathrm{~mm}$ from the endopelvic fascia, tumors with ingrowth in the internal sphincter or the levator ani muscle, and all cT4.

\section{Low and ultralow anterior resections Introduction}

Low anterior resection (LAR) or ultralow LAR (ULAR) can surgically treat rectal tumors, not compromising the sphincter complex. The findings from recent studies supporting the oncologic safety of a shorter distal margin of only $1 \mathrm{~cm}$ [28] when it is combined with multimodality treatment and clear radial margins have changed the previous recommendation DRM of at least 5 for rectal cancer resection, allowing to perform more sphincter-preserving surgeries $[69,70]$. The uLAR represents the complete resection of the rectum and mesorectum (total proctectomy), a technically ideal curative procedure when feasible.

\section{Patient selection}

Although TME is still considered the gold standard for all rectal cancers with good oncologic outcomes (5-year overall and disease-free survival of $93.2 \%$ and $79.7 \%$, respectively) [71]. LAR is performed for tumors in the middle and upper third of the rec- 
tum and occasionally for lesions in the lower third, generally located $>5 \mathrm{~cm}$ from the DL. The uLAR removes the rectum en bloc near the attachment point at the puborectalis for tumors located 1 to $2 \mathrm{~cm}$ above the DL [24].

Besides these criteria, upfront surgery can be an alternative for patients with T3N0 tumors $5 \mathrm{~cm}$ from the anal verge or T1-2N1 tumors. Inclusion criteria proposed for this option are negative MRI-predicted CRM, negative extramural vascular invasion, and $\mathrm{T} 3 \mathrm{a} / \mathrm{b}$ (extramural spread of $<5 \mathrm{~mm}$ ) as proposed by the MERCURY [72] and QuickSilver trials [73].

\section{Surgical technique}

For ULAR, in the distal part of TME to expose the pelvic floor and levator muscle, we have proposed the "gate approach" dissection technique for rectum mobilization [74]. The gate approach facilitates preserving the neurovascular bundle, located external to the junction of visceral fascia and Denonvilliers fascia (DVF), and identifying the middle rectal artery (MRA) when present. This step is included in the anterolateral dissection of TME, starting behind the DVF lateral side and detaching it from the mesorectal fascia [1]. The dissection progresses to the deep pelvic floor, making a clear gate between the mesorectal fascia and the pelvic floor. In addition to the deep posterior dissection, advancing in this surgical plane will leave the lateral ligament of the rectum (LLR) and the upper soft connective tissue layer hanging like a bridge between the parietal and mesorectal fascial, where the dissection can proceed with ligation of MRA if present while preserving the pelvic plexus within the parietal wall.

If possible, the rectum is transected after complete mobilization 1 to $2 \mathrm{~cm}$ distal to the tumor margin with a linear endo stapler, and a double-stapled anastomosis (DST) is performed. For lowlying tumors, 1 to $2 \mathrm{~cm}$ near the DL, a perianal stage of the procedure is done to expose the anal canal with self-retaining retractors. The lower margin of the tumor is identified under direct vision. Rectal transection is started at exactly $2 \mathrm{~cm}$ distal to the tumor margin $[75,76]$. The rectum's further dissection and mobilization are continued to the lateral and anterior wall through the same plane. After the circumferential transection of the rectum, the cut edge is closed with interrupted sutures to prevent cancer cell dissemination. The specimen can be extracted by the anus and the proximal colon is then drawn to the DL without creating tension and opened to perform an end-to-end CAA above the sphincter complex [76]. For hand-sewn CAA, we use a curved taper point 4-0 needle with absorbable thread to a layer-to-layer anastomosis (Fig. 9A). A pelvic suction drain is placed before closure, and a temporary derivative loop ileostomy is made closed 8 to 12 weeks after surgery.

\section{Postoperative complications}

In a comparative retrospective study, an anastomotic leak occurred in $8.1 \%$ and $1.3 \%$ of patients with TME and partial mesorectal excision, respectively $(\mathrm{P}<0.001)$, with a higher leakage rate in tumors
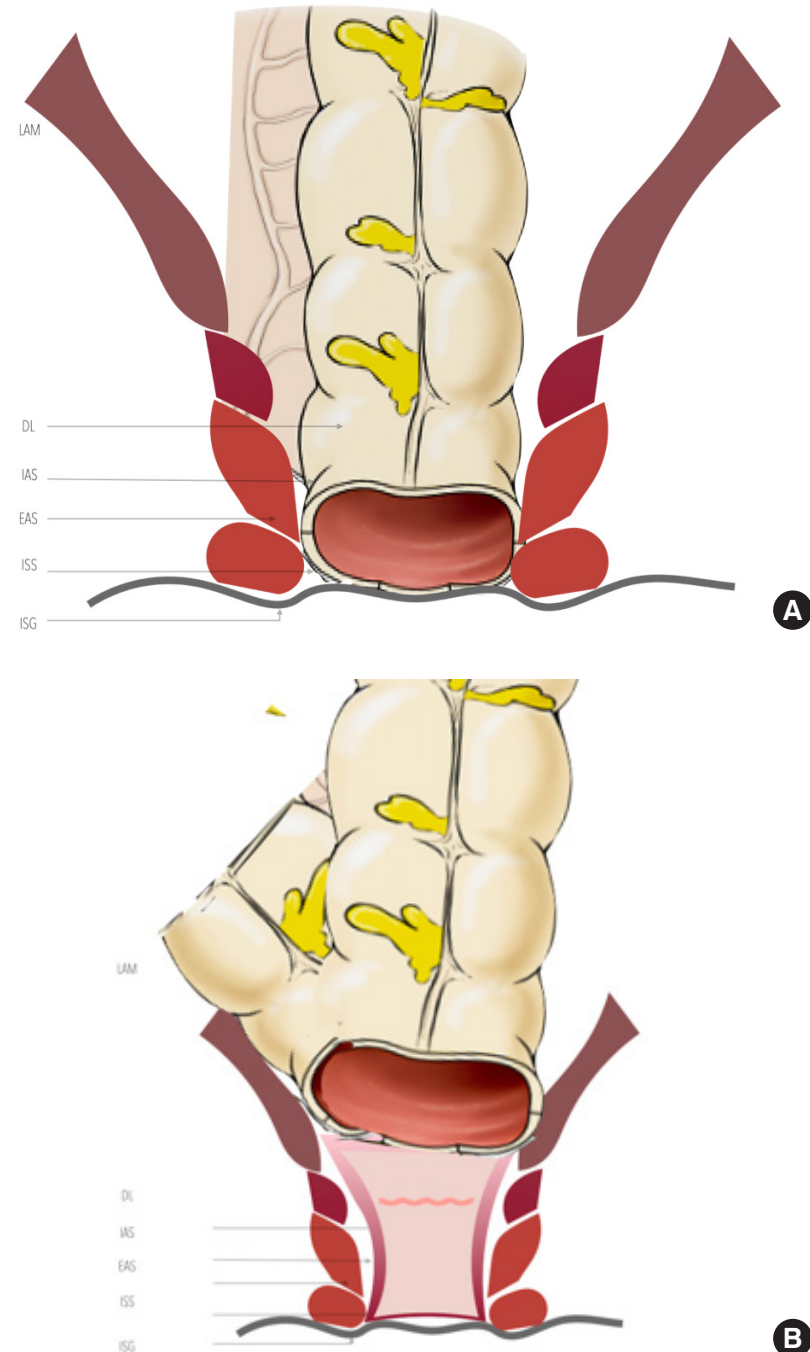

A

Fig. 9. An ultralow anterior resection. (B) After ultralow anterior resection, a hand-sewn anastomosis is usually preferred in the narrow pelvic cavity. (C) Baker-type or side-to-end coloanal anastomosis. LAM, levator ani muscle; DL, dentate line; IAS, internal anal sphincter; EAS, external anal sphincter; ISS, intersphincteric space; ISG, intersphincteric groove.

requiring a TME than in those higher tumors where partial mesorectal excision provides adequate mesorectal clearance [71, 77].

A Japanese retrospective multivariate multicenter analysis [52], found a significant relation with tumor size $(\geq 4 \mathrm{~cm})$ and tumor category (T4) as independent risk factors for postoperative complications, although pathologic resection margins were negative in all patients. In Korea, we found postoperative complications were associated with poorer oncologic outcomes and negatively impacted 5-year disease-free survival after laparoscopic LAR for rectal cancer [78]. The local recurrence rate in the complication group increases more than in the noncomplication group.

Late complications can be observed in $7 \%$ of cases, with a higher 
risk for patients who received NCRT. Late complications can manifest as fistula (39.7\%), chronic sinus (31.1\%), and stenosis (29.2\%) being symptomatic in $80 \%$ of cases and representing a complex situation for the colorectal surgeon.

\section{Oncologic outcomes}

Although initially, performing uLAR for low rectal cancer was a controversial choice, it has been proved that sphincter-saving surgeries do not affect the survival or recurrence after a curative resection for adenocarcinoma of the rectum at or below the peritoneal reflection when a uLAR is adequately performed with a $2-\mathrm{cm}$ DRM, achieving comparable local recurrence and survival rates similar to APR [13]. In the same way, the anastomosis techniques (DST or CAA) do not show any difference in disease-free and disease-specific survivals and the frequency and location of recurrence [79].

For cT1-2/N1, cT3N patients without CRM involvement nor lateral LN metastasis, upfront surgery without neoadjuvant therapy can be considered in early-stage patients with a good prognosis [80]. In our experience, oncologic outcomes of LAR and ULAR are significantly affected by positive ypN stage (hazard ratio [HR], 2.110; 95\% confidence interval [CI], 1.144-3.892; $\mathrm{P}=0.017$ ) and tumor regression grade of 3 or more (HR, 2.962; 95\% CI, 1.434$6.119 ; \mathrm{P}=0.003$ ) with a recurrence rate of $20.0 \%$ in our locally advanced rectal cancer patients, coinciding with National Comprehensive Cancer Network guidelines where a recurrence rate of $20.8 \%$ is reported [81]. The high incidence of distant metastasis of rectal cancer, especially lung metastasis, can be explained as hematogenous metastasis by bypassing the liver via direct venous drainage into the vena cava [81-83].

\section{Functional outcomes}

Most of the patients (approximately 90\%) who undergo SPS for low rectal develop LARS. The symptoms are diverse, including increased bowel movement frequency, urgency, fecal incontinence, sense of incomplete emptying, and fragmentation. The leading causes of LAR are presumed to be anal sphincter damage during the operation, reduced neorectal compliance as a reservoir, altered motility of the neorectum possibly caused by denervation during deep pelvic dissection, and NCRT [47]. It has been speculated that the long duration between the creation of protective ileostomy and its reversal could worsen neorectal compliance and lead to LARS; however, recent studies confirm it was not a significant independent predictor of post-closure complications rate [84].

To improve the reduced neorectal compliance as a reservoir after a coloanal or colorectal anastomosis, a multicenter prospective randomized study anastomosis aimed to evaluate the impact on QoL and bowel function when comparing colonic J-pouch (CJP) and straight colorectal reconstruction LAR, concluding that there is no significant difference between groups that support the routine use of CJP reconstruction [85].

As an alternative to CJP reconstruction, a side-to-end CAA
(SEA) or Baker type $[86,87]$ has been widely used as an effective anastomotic strategy to achieve similar postoperative bowel function without increasing the risk of complications compared with CJP anastomosis. In this technique, a DST anastomosis is performed, extracting the anvil on the free side of the proximal colon, $5 \mathrm{~cm}$ proximal to the stapled resection margin, and secured with a purse-string suture (Fig. 9B). Finally, the stapler is inserted transanally, and the SEA is completed [88]. A comparative metaanalysis was conducted by Hou et al. [89], finding that advantages of SEA include a shorter operating time, a higher anorectal rest pressure, higher defecation frequency 12 months after surgery, with no difference in postoperative complications and oncologic outcomes.

In terms of sexual and voiding functions, some studies have demonstrated the importance of pelvic autonomic nerve preservation during TME. A Dutch TME trial showed overall sexual dysfunction in $76 \%$ of male patients and $62 \%$ of female patients. Voiding dysfunctions, including urinary incontinence, retention, urgency, and incomplete voiding, are known to be less severe than sexual dysfunctions, and the incidence has been reported to be $>30 \%$ [47].

\section{Expert comments}

The uLAR represents one of the most common surgical procedures performed by colorectal surgeons for distal rectal cancer. Preoperatively, a thoughtful evaluation of the rectal MRI should determine any close relations between the tumor and the surgical dissection planes at the deep pelvis. Especial attention should be paid to the extent and location of the tumor, the relation between levator ani and the distal tumor border. These pieces of information should be kept in mind during the sharp anatomical dissection of the mesorectum, which should be performed circumferentially down to the pelvic floor.

Several intraoperative complications have been described for uLAR and LAR during the transanal or perineal approach. A cross-sectional study regarding surgical errors by junior fellows and trainees in low rectal cancer surgery has recently been published, with exciting conclusions [90]; in this cohort, inadvertent injuries were made during preparation for the DST CAA by either forceful introduction of the circular stapler, inadequate pursestring sutures or incidental opening of the stapled anorectal stump. Senior consultants successfully managed all injuries by colonanal hand-sewn anastomosis, colon pull-through, or combined abdominal and perineal approach. Studies like this recall the attention for specialized proctoring and structured training programs focused on developing competencies in young surgeons to overcome incidental surgical complications.

Regarding the coloanal reconstruction, in some instances, the CJP may not fit at deep narrow pelvis in male patients. Personally, we think CJP does not improve LARS; therefore, we prefer to use straight CAA, which shows acceptable functional outcomes with long-term improvement of the number of bowel movements per 
day and urgency or soilage events [91].

A novel technique for precision functional SPS has been proposed in Shanghai by Zhuang et al. [92] with the use of a newly transanal microsurgery system proposed by Zhuang et al. [93] to precisely resect rectal tumors and preserve sphincter function in ultralow rectal cancer patients. During the perineal stage of the surgery, a transparent screw-shaped anal dilator creates an open field to help in the measurement, localization, and resection of the lower edge of the tumor. The rectum is transected, and the specimen is taken out through an anoscope to avoid abdominal incision. A special device aids in supporting the proximal bowel end to perform a full-thickness anastomosis, and another special transanal tube (TAT) is placed afterward without a routine prophylactic stoma [94]. Although more studies need to be done to evaluate different long-term oncological and functional outcomes, this minimally invasive natural orifice specimen extraction technique promises to enhance surgical efficiency, reduce the need for prophylactic stoma and surgery-related costs with minor abdominal surgical incisions.

\section{Intersphincteric resection Introduction}

Schiessel et al. [95] introduced ISR in 1994 in Australia as a safe surgical treatment option for low-lying rectal cancer with good oncologic and functional outcomes. In Japan, in early 2000, a multicenter phase II clinical trial evaluated the safety and feasibility of ISR for low-lying rectal cancer in a total of 110 patients [96] being able to currently offer for low-lying $\mathrm{T} 1$ or $\mathrm{T} 2$ rectal cancer ISR as a standard surgical treatment option [96, 97]. A safety and feasibility study of laparoscopic ISR for low-lying rectal cancer demonstrated the favorable short-term outcomes on stage 0 and 1 low rectal cancer with complete preservation of pelvic autonomic plexus [98].

For ultralow rectal cancer, the absence of the mesorectum in the most distal portion is the key to the interest in removing the internal sphincter to widen the CRM unless the tumor had invaded the external sphincter $[33,99]$. A surgical dissection between the internal and external anal sphincter can be performed to resect the rectum en bloc with the IAS and the anal mucosa. This procedure combines the transabdominal with an anal approach and a hand-sewn CAA.

\section{Patient selection}

The resection line of the rectum or anal canal varies depending on the distance of the tumor from the anal verge and the extent of ISR (Fig. 10) [33, 99-101]. The technique variations include: partial ISR is defined as a $1 / 3$ resection of the upper part of the internal sphincter performed when there is enough distal margin above the DL starting the resection at this point, subtotal ISR as a $2 / 3$ resection of the internal sphincter when the distal edge of the tumor is $>2 \mathrm{~cm}$ from the DL, starting the resection between the DL and intersphincteric groove (ISG), and total ISR as a complete resection of the internal sphincter with resection from the ISG performed when the tumor has spread beyond the DL $[47,100]$.

\section{Surgical techniques}

The perineal dissection can be done as the first or second stage of operation regarding the surgeon's preference. An initial perineal dissection could help determine the extent of distal resection and guarantee a safe CRM. The technique begins with adequate exposure of the anal canal and anatomical landmarks (anococcygeal ligament, levator ani muscle, puborectalis, and DVF in males and rectovaginal septum or anterior dense tissue in females) by exposure of the anal canal with self-retaining retractors.

The anatomical landmark to start the procedure is the ISG or Hilton's line identified by digital rectal examination (Fig. 11 [102]). For hydrodissection and hemostasis, $0.25 \%$ bupivacaine mixed with epinephrine can be injected into the submucosa below the DL. A distal margin of resection of 1 to $2 \mathrm{~cm}$ long is determined under direct view. Circumferential incision of the mucosa is performed according to the appropriate ISR extent (partial, subtotal, or total). The rectum can be closed transanally by a
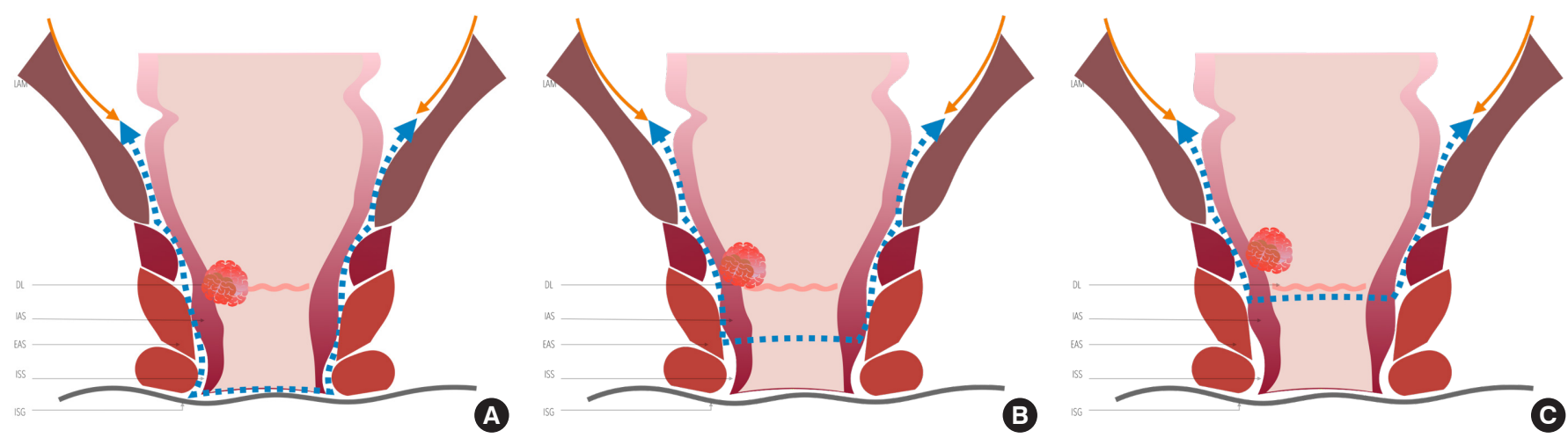

Fig. 10. Intersphincteric resection (ISR) is indicated to tumors located at the surgical anal canal and confined to the internal anal sphincter (IAS), not invading the levator ani muscle (LAM) or external anal sphincter (EAS). (A) Total ISR. (B) Subtotal ISR. (C) Partial ISR. DL, dentate line; ISS, intersphincteric space; ISG, intersphincteric groove. 

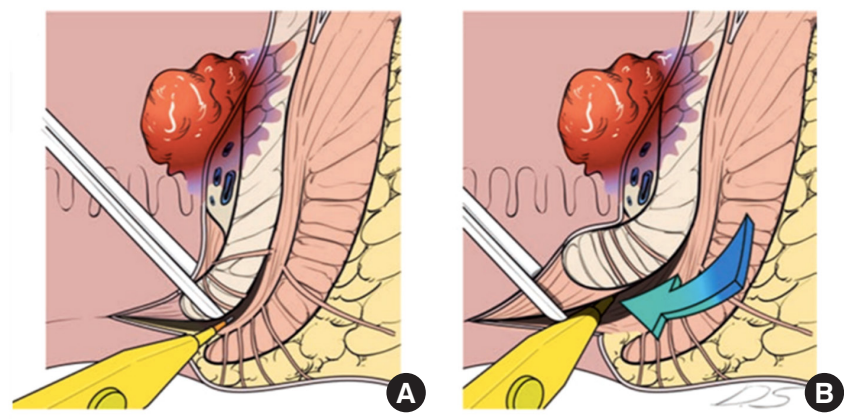

Fig. 11. Intersphincteric dissection begins in the intersphincteric groove or Hilton's line. Adapted from Kim et al. [102] with permission of Springer.

purse-string suture to avoid tumor seeding and prevent contamination of the intraluminal contents.

The intersphincteric plane is utilized for dissecting in an avascular plane, beginning with a posterior dissection, begins at the level of the DL for partial-ISR cases, between the DL and the ISG for subtotal-ISR cases, or at the ISG for total-ISR cases, identifying the anococcygeal ligament and a dense fibrous tissue composed of smooth muscles connecting the distal rectum to the coccyx [102]. Dissection progresses laterally, exposing the levator ani muscle and puborectalis, separating the lower part of the pelvic floor from the rectum. The muscular rectal wall is freed using cautery at the level of the anorectal ring, and division of the superior sheath of the pelvic floor and the presacral Waldeyer's fascia allows to reach the abdominal dissection [28]. Full mobilization is confirmed using the index finger. Careful anterior dissection should be performed to avoid injuries to the urethra anteriorly and the rectum posteriorly [103].

The rectum can then be extracted and resected through the anal canal or an abdominal incision. Care should be taken to avoid sphincter injury or tumor perforation during specimen extraction in a narrow pelvis, bulky mesorectum, or large tumors that can difficult the extraction.

After confirming adequate margin, the proximal rectum is transected. Stay sutures are taken on the left and right sides of the proximal colon. The mesocolon orientation is confirmed transabdominally to avoid twisting the anastomotic segment when the colon is delivered into the pelvic cavity, and anastomotic tension should be avoided. Adequate colonic length can be ensured by splenic flexure mobilization or high ligation of inferior mesenteric vein (IMV).

A DST anastomosis can be feasible in partial or subtotal ISR, and a manual purse-string suture is made around the anoderm to facilitate the use of the circular stapler. For total ISR, a hand-sewn CAA (Fig. 12 [102]) with a curved taper point 4-0 needle with absorbable thread full-thickness anastomosis. Finally, pelvic drains and a rectal tube are placed, and a protective loop ileostomy is performed [47].
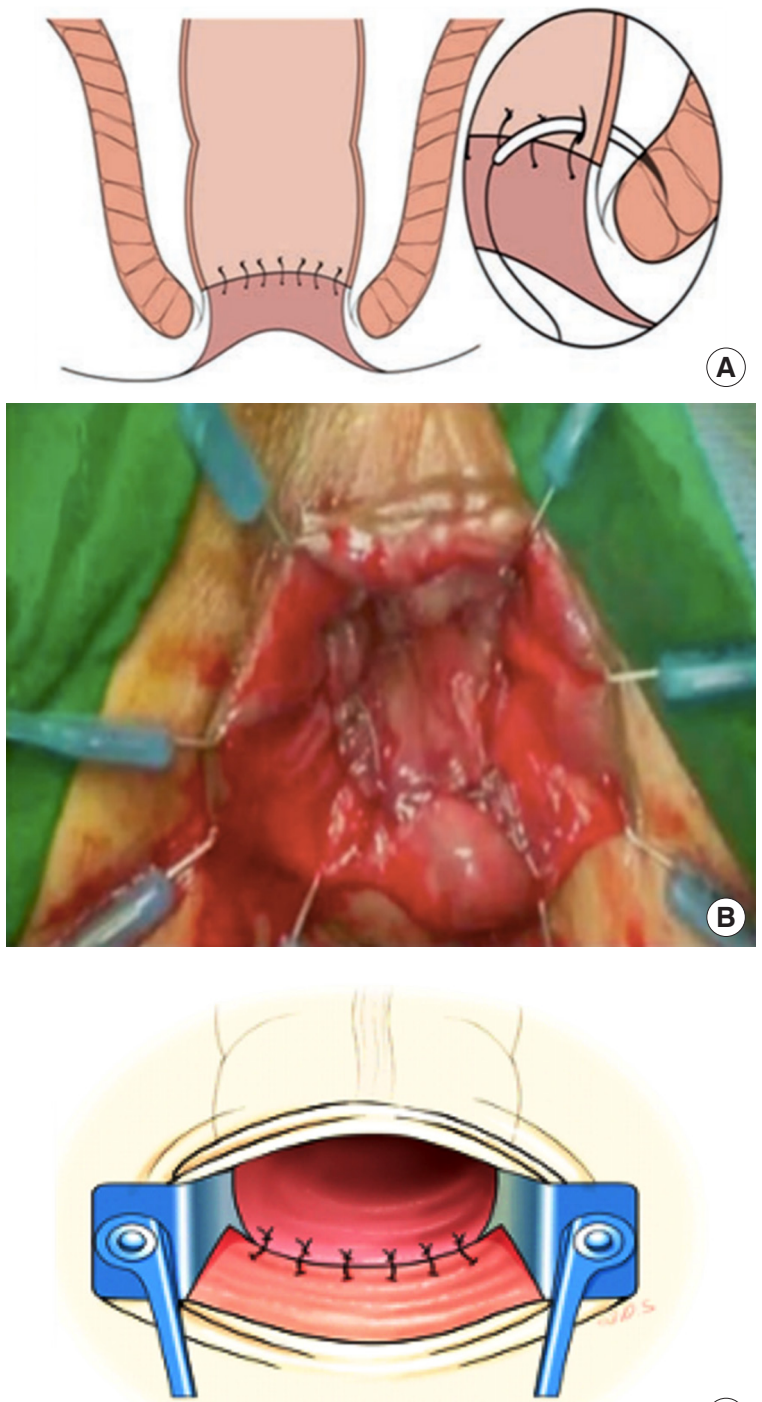

(C)

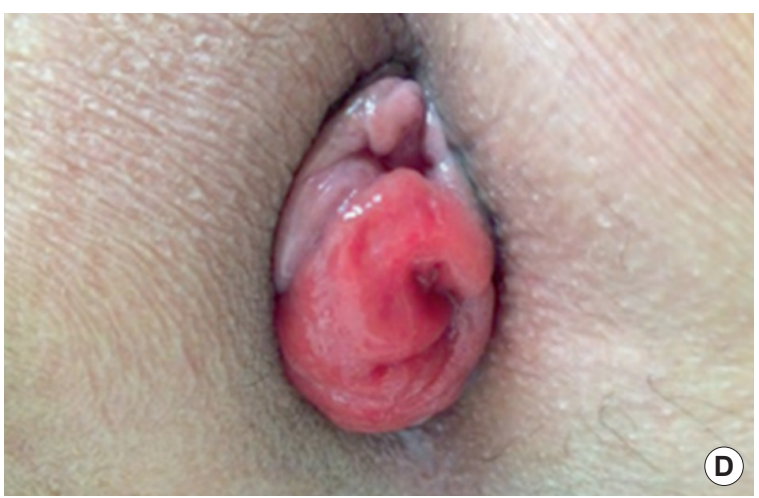

Fig. 12. (A) Hand-sewn coloanal anastomosis with interrupted absorbable sutures between the colon, anal mucosa, and external sphincter. (B, C) Colonanal anastomosis result. (D) Prominent mucosal prolapse after intersphincteric resection causing wet anus. Adapted from Kim et al. [102] with permission of Springer. 


\section{Postoperative complications}

ISR is not recommended for cases with poorly differentiated adenocarcinomas or preoperative compromise of anal continence, nor patients with tumor invasion to the external anal sphincter or the levator ani muscle [24]. Systematic reviews have reported an R0 resection rate of patients who underwent ISR was $97.0 \%$, the AL rate range of $4.3 \%$ to $9.1 \%$, and development of CAA strictures in $8.4 \%$ to $15.9 \%[24,47]$.

Anastomotic complications (leakage and stricture) have been identified as risk factors for permanent stoma after ISR for ultralow rectal cancer with a significant incidence of permanent stoma between the ISR groups (partial vs. subtotal vs. total, $8.3 \%$ vs. $20 \%$ vs. $25.8 \% ; \mathrm{P}=0.02$ ) and a 5 -year cumulative incidence of permanent stoma of $17.4 \%$ [104].

Mucosal prolapse at the site of anastomosis (Fig. 12D) have also been reported in several case series with an incidence of $4.6 \%$ to $5.9 \%$ [105-107] and diagnosed within a median of 6 months after partial or total ISR, with a higher incidence in female $(9.5 \%)$ than male (2.5\%) [108]. Mucosal prolapse is explained by Alessa et al. [106] due to increased intra-abdominal pressure postoperatively, a thin neorectal wall compared to the rectum, loss of IAS pressure, and the use of a redundant colon segment for CAA. Several treatment options have been described, as the Delorme procedure, redo of CAA by Altemeier procedure, or APR for severe incontinent patients $[105,107,108]$.

\section{Oncologic outcomes}

Recent evidence suggests that ISR is an oncologically acceptable surgical technique for low rectal cancer, similar to APR. In the original long-term cohort of Schiessel et al. [95], 6 patients (5.3\%) developed local recurrence, and 4 (3.5\%) died within a median follow-up time of 94 months [95]. Denost et al. [109] analyzed 303 patients who underwent ISR in a single institution for 25 years. The 5-year local recurrence was 3.5\% to $4.3 \%$, disease-free survival was $73 \%$. In our study, we reported 3 -year cumulative local recurrence was $9.1 \%$, and 3-year disease-free survival was $79.2 \%$ [91]. Similar results were reported in a Japanese study of 2,125 patients, with a 5 -year overall survival rate of $92.8 \%$ for stage I, $89.3 \%$ for stage II, and $73.6 \%$ for stage III, and the 5 -year cumulative local recurrence rate after ISR was $11.5 \%$ in a followup period of 58 months [97]. The systemic review by Akagi et al. [100] yielded satisfactory oncologic outcomes, with reported rates of $19.24 \%$ for overall recurrence, $2.5 \%$ to $19.0 \%$ of distant metastasis, and $0 \%$ to $22.7 \%$ for local recurrence rates. The survival rates were favorable with disease-free and overall 5-year survival rates ranging from $69 \%$ to $86 \%$ and $79 \%$ to $97 \%$, respectively [97].

The use of robotic systems has considerably influenced the oncological outcomes of the ISR technique in recent years. Studies comparing long-term feasibility between robotic ISR and laparoscopic ISR have demonstrated no significant differences in local recurrence, overall survival, or disease-free survival between the 2 techniques. Moreover, investigators have lauded the ergonomic efficiency of the robotic platform, with improved dexterity and visualization $[46,110]$.

\section{Functional outcomes}

After rectal cancer surgery, QoL faces 2 different challenges; between a good-functioning permanent stoma with a life-changing body image and the risk of a poor-functioning CAA.

The most critical point in preventing urinary and sexual dysfunction is to avoid injury to the autonomic nerves, including the superior and inferior hypogastric nerve plexus and neurovascular bundles going into genitourinary organs. Failure in bladder emptying is generally known to resolve within 3 months; however, symptoms persisting after 6 months are reported to be mostly permanent. Sexual dysfunction from partial injury of nerves is usually resolved within 3 to 6 months; however, delay in the treatment of sexual dysfunction might lead to permanent dysfunction [47].

As the extent of resection of the anal sphincter becomes wider, postoperative defecatory dysfunction (e.g., fecal incontinence) becomes a more severe problem, with $42 \%$ of patients experiencing major postoperative bowel dysfunction reported rates [109]. In particular, it has been reported that the incidence of defecatory dysfunction is high in patients who receive preoperative RT, those with AL, and the elderly [24].

We have compared ISR functional outcomes vs. uLAR for low rectal cancer in 146 patients [91] finding a higher incidence of major fecal incontinence in the ISR group (75.9\% vs. $49.3 \%$; $\mathrm{P}=0.016$ ), with similar overall survival and disease-free survival rates. The median Wexner score varied significantly between the groups; the score was lower in the ULAR group than in the ISR group (10 vs. $14, \mathrm{P}=0.043$ ).

The long-term results of ISR obtained by Yamada et al. [97] also showed that even though ISR achieved a reasonable survival rate with low mortality and morbidity, postoperative fecal incontinence was significantly high. Defecatory incontinence was present in $37.7 \%$ of cases, with no significant difference in bowel frequency according to the type of ISR performed. The incontinence rate of patients with straight CAA anastomosis was significantly higher than in those with a CJP or end-to-side reconstruction.

Nevertheless, evacuation disorders such as inability to get to the toilet in time (86.5\%), sense of incomplete evacuation (85.4\%), and having another bowel movement within 15 minutes from the previous 1 (81.3\%) also constipation, dyschezia, need for enemas, and other symptoms were reported in both studies, and although not being statistically significant represents a factor compromising the patients QoL $[91,97]$.

A review by Park and Kim [111] summarized important insights about the possible reasons for poor long-term functional outcomes and compromised QoL in some ISR cases. Besides the deleterious influences in the anorectal function of NCRT, continence is mainly compromised by the partial or complete removal of the IAS, with a subsequent fall in the resting anal pressure. This 
anal sphincter insufficiency accompanied with neorectal insufficiency by straight CAA limits the tolerable rectal volume, clinically expressed with tenesmus, urgency, and anal incontinence (with rates of major incontinence ranging from $0 \%$ to $25.8 \%$ after an ISR). Anorectal compliance might be improved by reservoirtype anastomosis like CJP or Baker side-to-end anastomosis lowering the risk of LARS and improving QoL in patients with internal sphincter resection.

\section{Expert comments}

Understanding the anorectal sphincter complex and surrounding neurovascular structures is essential for optimal oncologic and functional outcomes in distal rectal cancer surgery. The anatomi$\mathrm{cal}$ anal canal is defined as beginning at the DL ending at the anal verge. On the other hand, the surgical anal canal is between the anorectal ring and the anal verge. The anorectal ring is usually palpable as a tight ring structure, and when patients are asked to squeeze the anal sphincter, it moves anteriorly. The anorectal ring consists of the external anal sphincter and the puborectalis muscles and is a good landmark for locating the tumor or the level of colorectal anastomosis. The anal canal has a cylindrical doublelayered shape. The inner layer is composed of the IAS and the conjoined longitudinal muscle, innervated by the autonomic nervous system (Fig. 13 [112]). In contrast, the outer layer is composed of the puborectalis muscle and external anal sphincter stimulated by somatic nerves. The groove between the internal and the external anal sphincter can be easily palpated and will be a landmark for ISR for low-lying rectal cancer; the ISG $[1,3,11]$.

We have observed that some patients who underwent ISR without radiation showed excellent functional outcomes, which we am surprised to hear. We would like to address the functional role
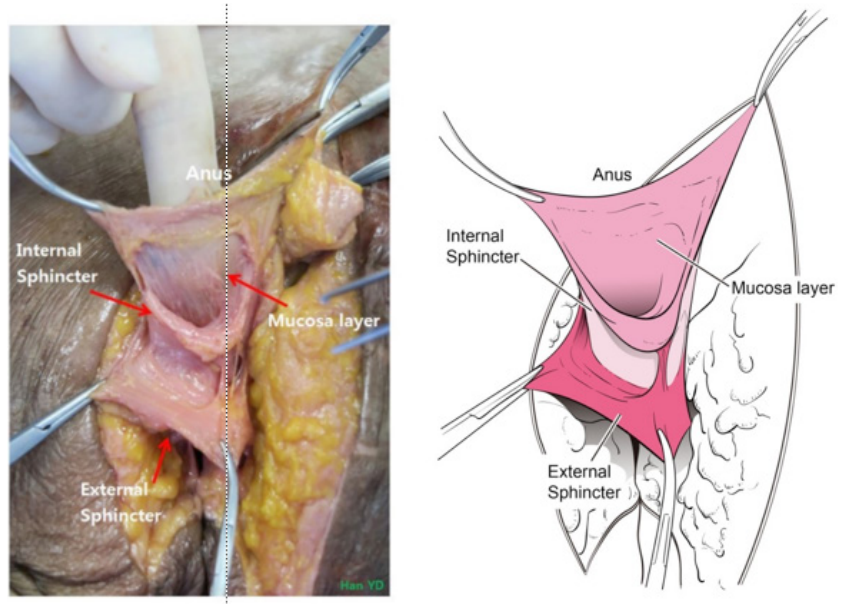

Fig. 13. Cadaveric dissection shows a clear cleavage plane between the internal and external anal sphincter. of conjoined longitudinal muscle. Of course, the portion of removal of IAS affects the functional outcomes, and the intersphincteric approach will destroy this muscle structure, which may impact the anorectal function.

We recommend reading the article written by Tsukada et al. [113], "Topographic anatomy of the anal sphincter complex and levator ani muscle as it relates to intersphincteric resection for very low rectal disease," where they describe that the length of attachment of the longitudinal muscle to the levator ani muscle is longer in the anterolateral portion and shorter in the posterior portion of the anal canal (Fig. 14 [114]). Usually, the ISR technique included the IAS and conjoined longitudinal muscle in the specimen, dividing it from the levator ani muscle, either by the transabdominal or perineal approach. The longest attachment area was the lateral part (between longitudinal muscle and levator ani muscle) based on this study.

Technically, we prefer the transabdominal approach, dissecting to the anal hiatus at the level of the pelvic floor, and a second time for the perineal approach where a circumferential incision is made on the intersphincteric grove, and dissection is circumferentially done, starting posteriorly. This way, the surgeon's dissection will make easy progress anterolaterally and then anteriorly, being careful not to damage the rectourethralis muscle, vagina, or other urogenital structures. For optimal functional results, it is crucial to select the right patient.

\section{Partial excision of levator ani muscle Introduction}

We have recently introduced the PELM technique followed by ISR and CAA to preserve the anal sphincter function and obtain oncologic outcomes while avoiding permanent colostomy in tu-

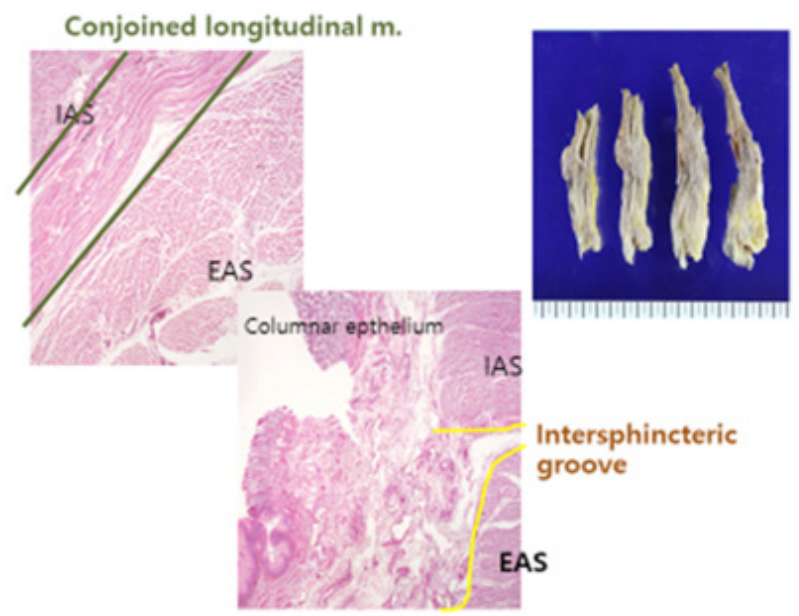

Fig. 14. Histologic studies $(H \& E$ stain, $\times 10)$ showed the internal anal sphincter (IAS, smooth muscle) and external anal sphincter muscle (EAS, skeletal muscle), along with the conjoined longitudinal muscle between them. Adapted from Lee et al. [114] according to the Creative Commons License. 
mors invading one side of the levator ani [34]. A unilateral excision of the levator ani and deep part of the external sphincter through the intersphincteric plane is slightly more advanced than ISR alone and not as wide as APR (Fig. 15 [115]) [116].

\section{Patient selection}

Similar to the ISR concept, PELM achieves a good CRM for tumors at the level of the anorectal junction performing the dissection and excision of the levator ani muscle compromised through the intersphincteric plane without compromising anal function [47]. Significant downstaging and downsizing of tumors after NCRT has facilitated resections permitting SPS and CAA for patients who would otherwise not have been candidates for this type of surgery [116].

Inclusion criteria for patients who can benefit from this technique are those with tumors located at the level of the anorectal ring, unilaterally invading or abutting the ipsilateral levator ani muscle, and intact external anal sphincter or tumors $[47,117]$.

\section{Surgical technique}

Initially, TME is performed classically during the abdominal phase in this technique, followed by dissection toward the levator ani muscles. Perineal dissection begins with anal exposure and a circumferential incision in the ISG, advancing the dissection in the intersphincteric avascular plane. On the tumor side, dissection advances diagonally to include de deep portion of the external anal sphincter and the ipsilateral levator ani muscle before entering the pelvis; this dissection is performed between the internal and external anal sphincter to the level about 0.5 to $1 \mathrm{~cm}$ below the tumor, where the direction of the dissection turned transversally to include the deep part of the external sphincter until the is-

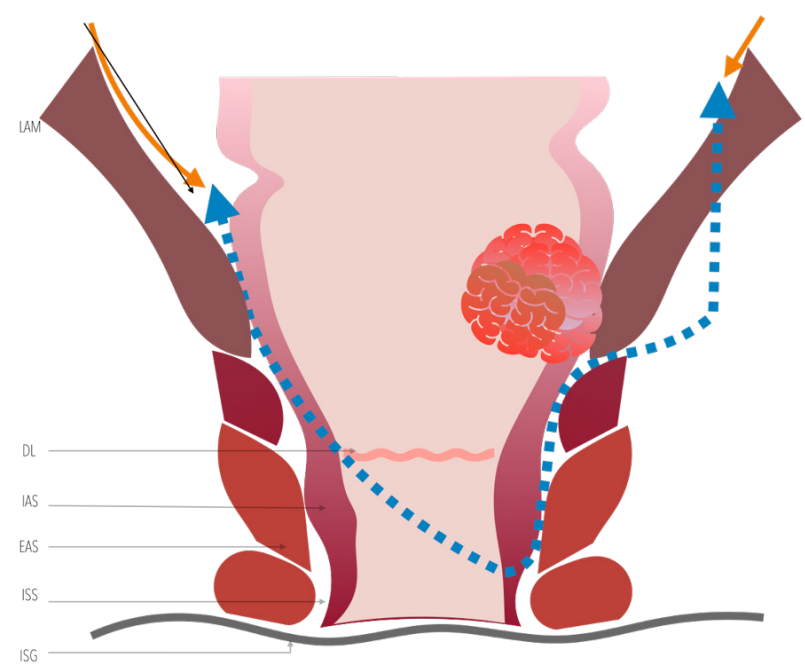

Fig. 15. Partial excision of levator ani muscle (LAM). DL, dentate line; IAS, internal anal sphincter; EAS, external anal sphincter; ISS, intersphincteric space; ISG, intersphincteric groove. Adapted from Yang et al. [115] with permission of Wiley. chiorectal fossa fat could be visualized [117]. On the opposite side, dissection progresses in a cephalad direction through the intersphincteric plane, preserving the ipsilateral sphincter complex. The specimen is extracted transanal, and a hand-sewn CAA and a protective ileostomy are performed [34, 47]. It is essential to recall that resection and anastomosis are made diagonal due to the asymmetrical resection of the distal rectum (Figs. 16, 17).

\section{Postoperative and oncologic outcomes}

In a case series of 23 consecutive patients who underwent robotic PELM for low rectal cancer at the anorectal ring level invading or abutting the ipsilateral levator ani muscle, the PELM technique demonstrated favorable postoperative surgical outcomes with 1 local recurrence at the anastomotic site 4 months after surgery and 2 systemic recurrences in the lung and liver at 25 and 6 months after surgery, respectively. During a median follow-up of 44 months, the 3-year local recurrence rate was $14.4 \%$. In terms of functional outcomes, incontinence was present in 2 patients with
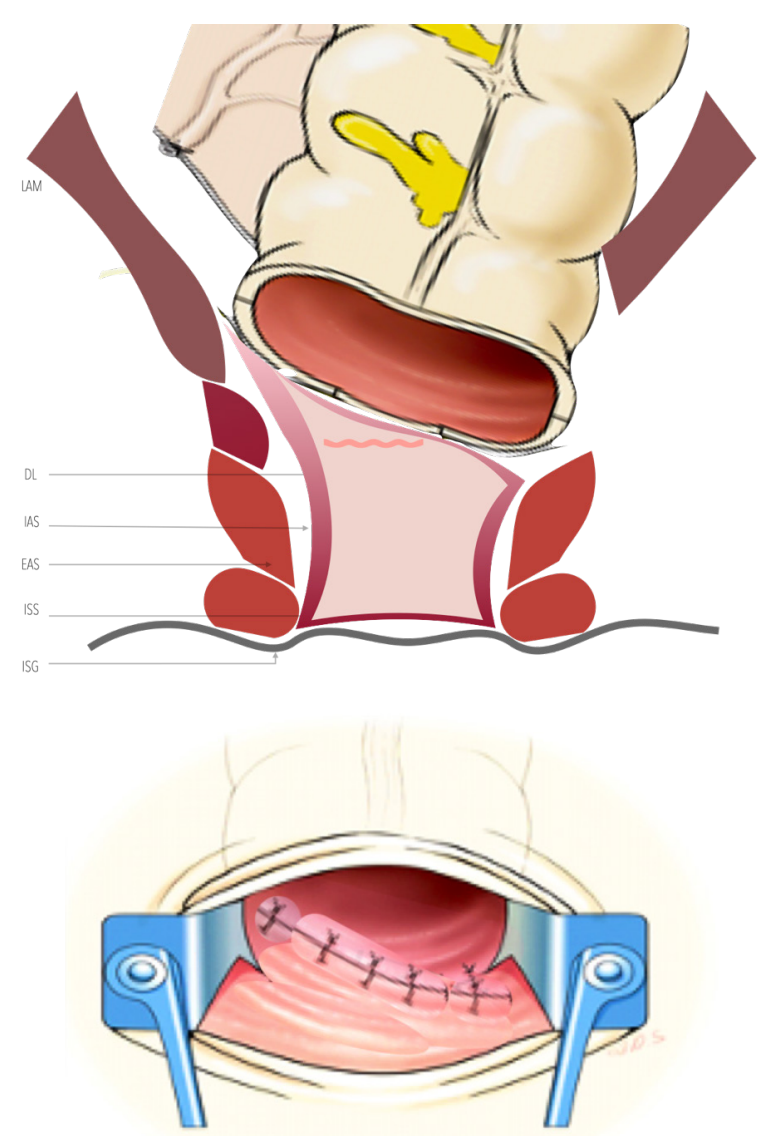

Fig. 16. Diagonal coloanal anastomosis for coloanal anastomosis for partial excision of the levator ani muscle. DL, dentate line; IAS, internal anal sphincter; EAS, external anal sphincter; ISS, intersphincteric space; ISG, intersphincteric groove. 
an average mean Wexner score of 9.4 for 6 of 13 patients who underwent ileostomy reversal (Fig. 18) [47].

\section{Expert comments}

NCRT followed by robotic PELM with ISR and CAA may provide increased opportunity to preserve the anal sphincter in patients with tumors invading the ipsilateral levator ani muscle at the level of the anorectal ring. Decades ago, these cases had been treated with APR and a permanent colostomy. Based on our cadaveric studies, the levator ani muscle is attached directly to the lateral surface of the longitudinal smooth muscle of the rectum.

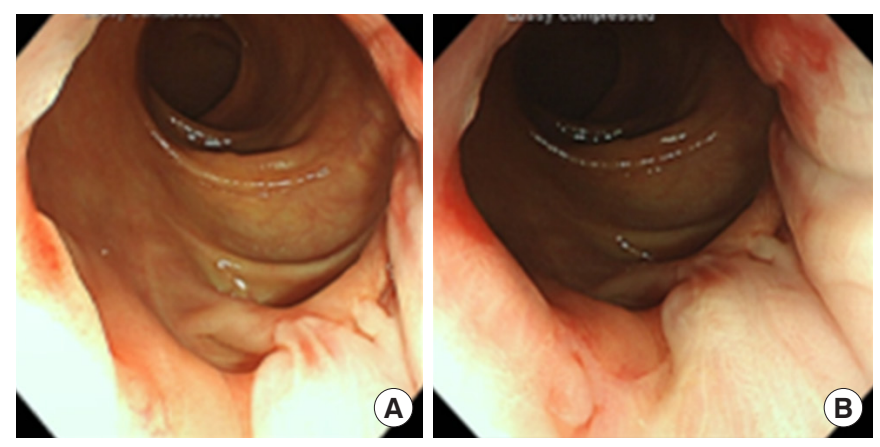

Fig. 17. Colonoscopy findings showed a diagonal anastomotic line in a patient who underwent partial excision of the levator ani muscle after preoperative chemoradiotherapy. A rectal exam can also confirm this oblique line.
Our microscopic findings at the level of the anorectal ring showed that the levator ani muscle partially overlaps with the external anal sphincter, as smooth and skeletal muscles intermingled with each other (Figs. 19 [115], 20 [117]).

PELM is a new surgical alternative to APR for low rectal cancer invading the ipsilateral levator ani muscle at the level of the anorectal ring. This procedure can be maximized when combined with robotic surgery. However, this technique needs longer follow-up to validate the oncological and functional safety.

\section{Abdominoperineal resection and extralevator abdominoperineal excision Introduction}

APR, or Miles operation, has been the gold standard treatment for low rectal cancer since 1907 [99]. Nevertheless, the high risk of specimen wasting and high rates of local recurrence and perineal wound complications lead to a more radical procedure by applying an extended posterior perineal approach by Shihab et al. [118], which resulted in a cylindrical specimen and avoided the "waisting" associated with conventional APR.

\section{Patient selection}

The ELAPE is explicitly indicated for low rectal tumors involving the levator complex whom MRI showed a T3 or T4 tumor located within $6 \mathrm{~cm}$ from the anal verge or those with fixed and tethered tumors on rectal examination and poor response to preoperative chemoradiotherapy infiltrating the external sphincter or levator muscles [119].

\section{- Defecography, 1 year after ileostomy closure}
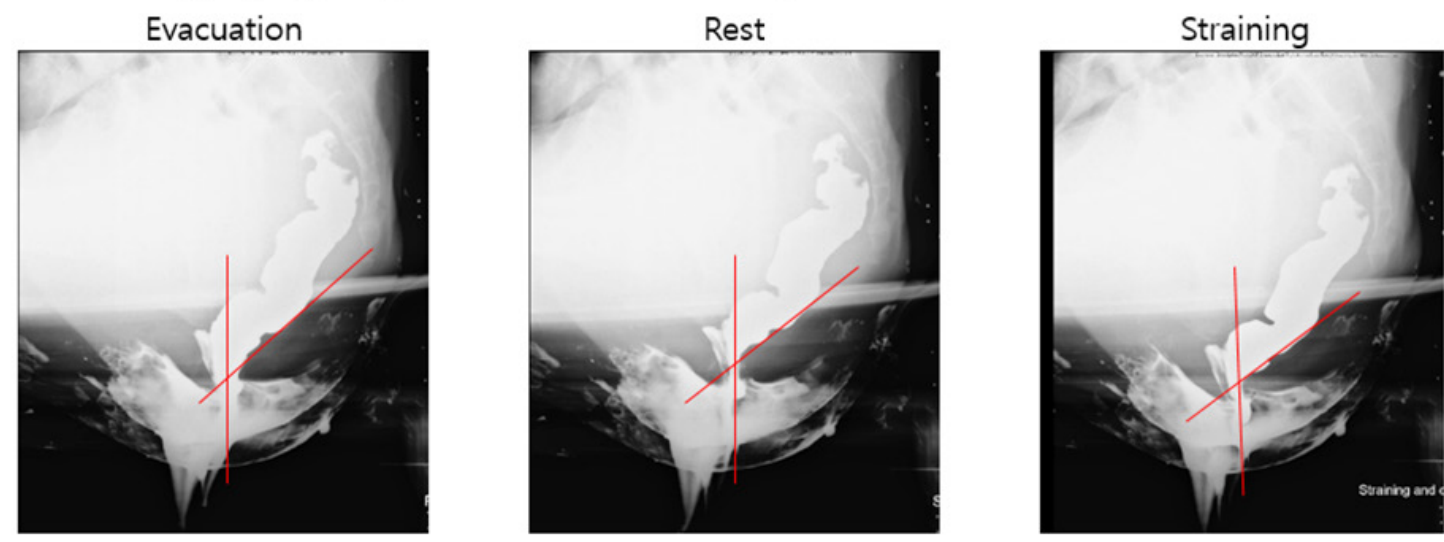

\begin{tabular}{|cc|}
\hline Anorectal angle & \\
\hline Evacuation & 140 \\
\hline Rest & 138 \\
\hline Straining & 127 \\
\hline
\end{tabular}

Fig. 18. Conventional defecography showed an acceptable anorectal angle oner after ileostomy closure in a patient who underwent partial excision of the levator ani muscle. 

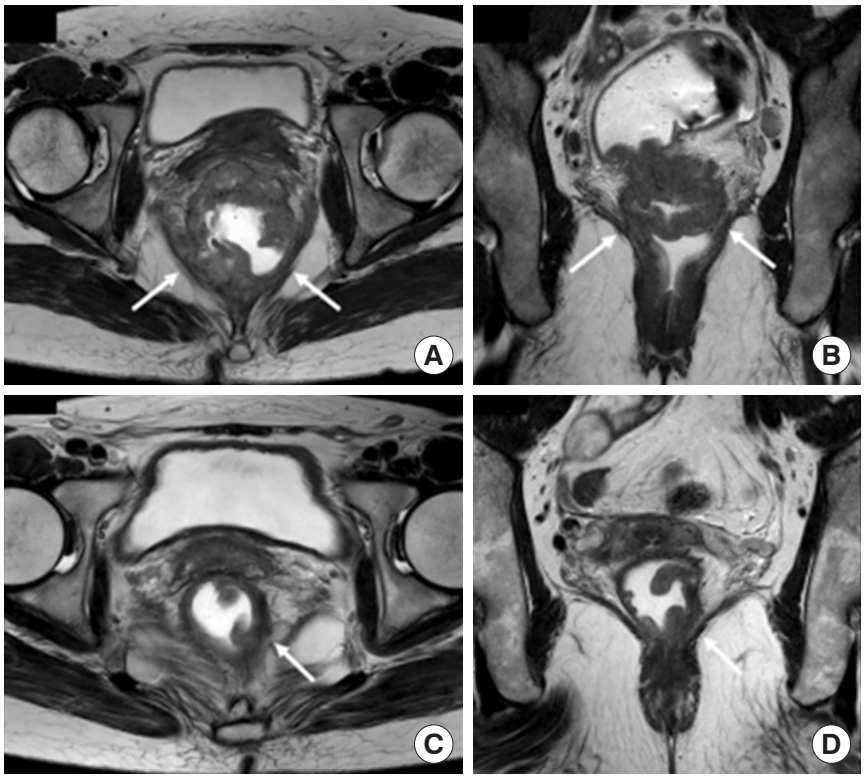

Fig. 19. (A, B) Rectal magnetic resonance imaging showed tumor invaded to the levator ani muscle bilateral extensively to be a candidate for extralevator abdominoperineal resection. (C, D) However, it showed that the tumor invaded only partly to the levator ani muscle so that it can be a candidate for partial excision of the levator ani muscle. Adapted from Yang et al. [115] with permission of Wiley.

\section{Surgical technique}

A purse-string suture is used to close the anus to begin the procedure. Anatomic landmarks are identified (ischiorectal fossae laterally, the perineal body anteriorly, and the coccyx posteriorly) to delimit the elliptical incision and progress the circumferential dissection. The ELAPE technique closely resembles Miles' original APR operation, which involves careful mobilization of the mesorectum up to the level of the levator muscles; however, in this approach, the dissection is continued along the outer surface of the levator muscles proximally until the insertion onto the pelvic sidewall, leaving the levators attached to the mesorectum and creating a cylindrical specimen with more tissue covering the tumor in the distal rectum. In the traditional APR, the dissection plane is outside the external sphincter muscle and progresses cephalad through the levator ani.

Critical points during the circumferential dissection are the anococcygeal ligament and the coccyx that might be disarticulated from the sacrum and removed along with the specimen to obtain an appropriate margin. Posteriorly, the dissection plane should stay in the avascular plane to avoid inadvertent vascular injuries to the presacral venous plexus or the internal iliac vessels. Anteriorly, dissection should be done carefully to protect the vagina in females and seminal vesicles/prostate in males, in addition to the urethra and trigone muscle of the urinary bladder [1].

The perineal phase of the procedure can be executed with the patient in a prone jackknife or lithotomy position $[118,120]$. The
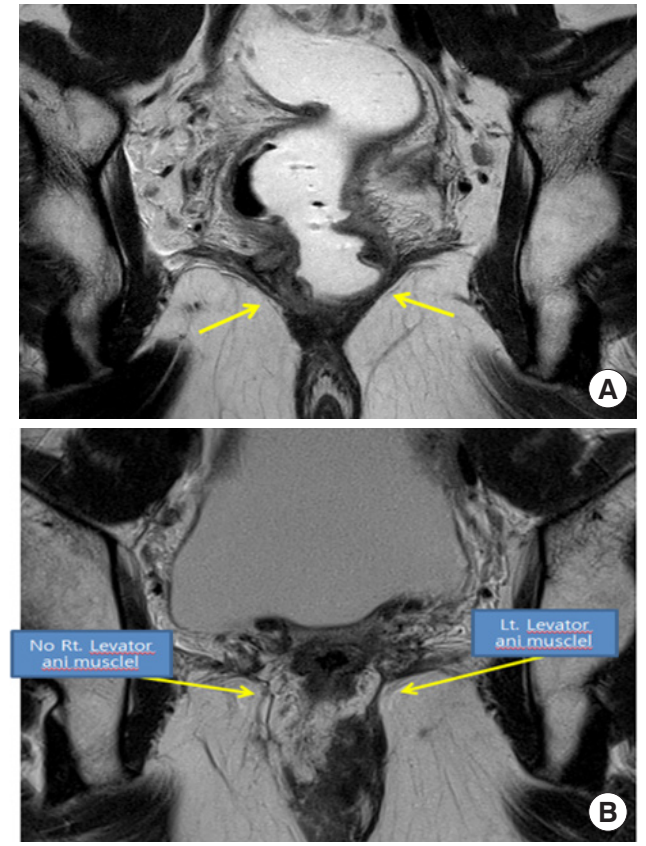

Fig. 20. Comparison between preoperative (A) and postoperative (B) rectal magnetic resonance imaging showed an absence of right-side levator ani muscle plate. Adapted from Noh et al. [117] according to the Creative Commons License.

prone jackknife position provides better visualization by allowing the rectum to descend outward after opening the pelvis, thereby revealing the plane between the rectum and the adjacent organs and preventing inadvertent tumor perforation $[17,121,122]$. Although the prone approach for APR is associated with decreased operative time and more precise perineal dissection with greater exposure, surgical positioning does not appear to make a difference regarding perineal wound infections, intraoperative perforation of the rectum, CRM positivity, or 5-year local recurrence [123]. Neither prone nor lithotomy position for ELAPE or APR have presented significant differences regarding bladder dysfunction, or sexual dysfunction, resulting from nerve damage [122]. Although some studies have suggested that in the prone jackknife position, the membranous portion of the urethra is more susceptible to injury and that a change in position might increase operating time and the risks of cardiac arrest or severe acute kidney failure [124].

Regarding the perineal wound closure, 2 recent metanalyses compared the outcomes of primary perineal closure with those of biological mesh reconstruction, finding no significant difference in the overall early wound complications rate, wound infection, or dehiscence rates, and chronic sinus or pain after primary closure or biological mesh reconstruction. After 1 year, the incidence of perineal hernia was significantly high after primary closure of the perineal wounds (odds ratio, 0.400; 95\% CI, 0.240-0.665; P= $0.001)$. Both studies found that the perineal hernia rate was sig- 
nificantly lower in biologic mesh reconstruction as compared to primary closure and the operation time and hospital stay were shorter after primary perineal closure $[125,126]$. Other studies have shown a lower incidence of surgical site infection using prophylactic negative-pressure wound therapy to prevent wound-related complications in surgery, with a risk reduction of about $25 \%$ to $30 \%$ [127].

\section{Postoperative complications}

Compared with traditional APR, the ELAPE was associated with a reduction in the rate of intraoperative perforation, lower rate of intraoperative perforation, and local recurrence, without any increase in the CRM positivity and postoperative perineal wound complication rate when compared with traditional APR in the surgical treatment of low rectal cancer $[128,129]$.

Although Habr-Gama et al. [130] reported that wound dehiscence is less likely to occur after ELAPE, because the ELAPE procedure has a better field of view and more precise homeostasis than APR, this technique might result in a significant perineal defect which needs reconstruction by a flap or biological mesh with increased perineal morbidity in the short- and longer-term [131]. A single-center retrospective study analyzed complications in ELAPE and APR with perineal wound complications present in $42 \%$ of patients who underwent ELAPE compared to $34 \%$ who underwent conventional APR. Among the patients who underwent ELAPE, 19\% had wound infection, 19\% had wound dehiscence, and $4 \%$ had flap loss [132]. In Sweden, a cross-sectional study evaluated 545 patients through the Swedish Colorectal Cancer Registry to investigate the prevalence of perineal symptoms 3 years after APR or ELAPE [131].

Perineal symptoms occurred in $50 \%$ of all patients. Delayed healing of the perineal wound ( $>4$ weeks) occurred more frequently after extralevator ELAPE than after conventional APR ( $32 \%$ vs. $11 \%, \mathrm{P}<0.001$ ), delayed healing was associated with an increased risk of more severe perineal symptoms (pain, sitting disability, paraesthesia, perineal tension, sensation of tingling/ stinging between, and perineal cramps or sensation of urgency).

\section{Oncologic outcomes}

West et al. [133] demonstrated the oncologic superiority of the ELAPE technique in a retrospective study by comparing specimens obtained from ELAPE to those taken using the conventional APR technique. The ELAPE technique cleared away more tissue at the distal rectum, which consequently resulted in lower rates of CRM involvement ( $14.8 \%$ vs. $40.6 \%, \mathrm{P}=0.013)$ and intraoperative perforations $(3.7 \%$ vs. $22.8 \%, \mathrm{P}=0.0255)$ than conventional APR. Kasai et al. [134] analyzed single-center experiences about APR. The patients received either the robot or laparoscopic approach. They found the incidence of urinary dysfunction and perineal wound infection were lower, and length of hospital stay showed shorter in the robotic approach.

Recently, Matsuda et al. [135] reported the feasibility and safety of transperineal minimal invasive APR for low rectal cancer to avoid the positive CRM and autonomic nerve injuries associated with APR. This technique adapts TaTME by performing the perineal phase of APR using a transanal single-port device and laparoscopic instruments for better visualization and dissection. In a 25 cases series, they concluded this approach seems superior to conventional laparoscopic APR in terms of short-term outcomes, with significantly lower rates of urinary disturbance and perineal wound infection with similar pathological outcomes and no local recurrences in 18-month follow-up.

\section{Expert comments}

We would like to raise the attention to a couple of issues about APR. One of the main reasons APR had a poor prognosis was a high rate of CRM+. Therefore, when distal rectal cancer is encountered with invasion to the levator ani, we try to excise the levator to avoid $\mathrm{CRM}+$, so ELAPE should be considered to obtain a sufficient CRM. For this technique, knowledge of the pelvic floor's anatomy is essential. The en bloc resection of the pelvic floor and the mesorectal fascia is essential to get a cylindrically shaped specimen. Unless the anatomy of the pelvic floor is fully understood, the excision of the levator ani muscle at its origin could be challenging and lead to an inadequate ELAPE. The levator ani muscle is more expanded and more visible in the jackknife position, and some surgeons insist that a jackknife position is necessary during this stage of the procedure. However, a transabdominal division of the extralevator through a robotic approach of the pelvic floor after robotic TME in Lloyd-Davies position is also an excellent way to perform ELAPE, facilitating the subsequent perineal dissection in the lithotomy position.

\section{DEBATED ISSUES}

\section{Pelvic lateral lymph node dissection Introduction}

TME is the gold standard treatment of mid and low rectal cancer, but PLND has been suggested as an approach to decrease recurrence and improve survival [136]. The high incidence of pelvic node metastasis is currently a critical issue in the management of low rectal cancer, creating controversies in the concept of regional vs. distant dissemination of the disease once it has already escaped the confines of the CRM and involved the lateral pelvic LNs (LPLNs; obturator, internal iliac, external iliac, and/or common iliac basin) [47].

\section{Patient selection}

In most Western centers, abnormal LPLNs were initially treated with standard NCRT and assumed to be sterilized by mostly extended beam radiotherapy to include the lateral LNs (LLNs) basins, followed by TME without PLND [136]. Nevertheless, the incidence of enlarged LPLNs remains high after NCRT. According to the Japanese guidelines, PLND is mandatory when the lower 
border of the tumor is located distal to the peritoneal reflection, and the tumor has invaded beyond the muscularis propria [24]. Regarding the size criteria, the definition of abnormal LPLNs varies between a short axis of 5 to $10 \mathrm{~mm}$ [137]. Pre-NCRT LLNs of $>5 \mathrm{~mm}$ short axis are present in $16 \%$ to $23 \%$ of patients with a primary locally advanced low rectal cancer. Although the prognosis of these cases is poor, in general, $40 \%$ to $50 \%$ of patients with R0 resection reportedly achieved 5-year survival [137].

A multinational, multicenter study recently reported that the 5 -year lateral pelvic wall recurrence rate was $19.5 \%$ in patients with an LPLN size of $>7 \mathrm{~mm}$ on preoperative MRI who were treated with TME only. However, the addition of selective PLND could significantly reduce the recurrence rate to $5.7 \%$, suggesting that TME after NCRT may be insufficient in patients with enlarged LPLN and that selective PLND may reduce local recurrence in this group $[138,139]$.

The criteria for cases where lateral $\mathrm{LN}$ dissection can be omitted are unclear. On the other hand, direct-to-surgery rectal resection with PLND is a treatment strategy commonly employed in Japan to improve oncological outcomes for rectal cancer was associated with worse local and total recurrence [24]. Nevertheless, a recent comparative study of patients with low rectal cancer who underwent direct-to-surgery resection and PLND vs. those who underwent TME alone found no difference in 5-year overall survival rate or 5 -year disease-free survival rate [140].

\section{Surgical technique}

PLND is a technically complex procedure [141]. After TME is performed, the ureter and hypogastric nerve are secured to establish the inner border of dissection. Starting from the iliac artery bifurcation, dissection proceeds along the external iliac artery and the external iliac vein until the major psoas muscle is reached. Once the presence of the obturator nerve, obturator vein, and obturator artery is confirmed, nerve-sparing dissection proceeds in a caudal direction, with the dissection along the obturator artery progressing from its origin toward the periphery to allow complete extirpation of the obturator LN. After removing the obturator LN, dissection of the internal iliac LN begins and is carried out until the Alcock canal area to ensure completeness. The lymphadenectomy is concluded with the en bloc removal of the tumor and suspicious LN specimen [138].

\section{Postoperative complications}

According to a recent meta-analysis, PLND is associated with longer operation time and increased complication rate [136]. The median operation time for PLND+TME was significantly longer than TME alone (360 minutes vs. 294.7 minutes, $\mathrm{P}=0.02$ ). PLND+TME was associated with higher odds of overall complications and urinary dysfunction than TME alone. Both groups had similar rates of male sexual dysfunction, AL, local recurrence, distant metastasis, overall survival, and disease-free survival. PLND also represents a high incidence of complications with a risk of urinary dysfunction and a higher possibility of sexual dysfunction, especially when PLND is performed in overweight patients [142, 143]. However, different opinions from Japan also suggested that PLND did not increase the risk of urinary nor sexual dysfunctions, stating that the risk of urinary dysfunctions was related to the blood loss and the tumor location and sexual dysfunctions were associated with the age of patients $[144,145]$.

\section{Oncologic outcomes}

A propensity score matching analysis of $\mathrm{pT} 3 / \mathrm{T} 4$ lower rectal cancer cases in the Japanese Society for Cancer of the Colon and Rectum (JSCCR) colorectal cancer registry from 1995 to 2004 also showed that the 5 -year overall survival rate of patients with lateral $\mathrm{LN}$ dissection was better than that of those without dissection (68.9\% vs. 62.0\%) [137]. Thus, even in cases in which preoperative chemoradiotherapy is performed, the omission of lateral $\mathrm{LN}$ dissection is not recommended.

Regarding the clinical value of lateral $\mathrm{LN}$ dissection in cases without evident lateral LN metastasis, the JCOG0212 study examined the noninferiority of the mesorectal excision alone to the mesorectal excision with PLND (ME+PLND) with the primary endpoints of relapse-free survival [146]. This study was conducted for patients with no lateral LNs with a short-axis diameter of $>10 \mathrm{~mm}$ on preoperative CT or MRI and whose tumor was located in the rectum, with the lower tumor margin below the peritoneal reflection. The frequency of local recurrence in the TME+PLND group was significantly lower than that in the TME alone group (7.4\% vs. $12.6 \%)$ without significant differences in the overall survival rate or local recurrence-free survival rate.

Kusters et al. $[69,147]$ also compared the oncological outcomes of low rectal cancer treatment between Japan and the Netherland. They reported a 5-year local recurrence rate of $6.9 \%$ in the Japanese TME+PLND group and 5.8\% in Dutch TME with NCRT group, with recurrence rates in the lateral pelvis of $2.2 \%$ and $27 \%$, respectively. These results suggest that NCRT and PLND may result in similar oncologic outcomes.

Assuming the NCRT can sterilize most LPLN metastasis based on previous literature, NCRT+TME offers a similar local control to TME+PLND with $5 \%$ to $10 \%$ of 5 -year local recurrence rates in advanced rectal cancer.

PLND, in addition to NCRT, may improve locoregional control in Western patients with low rectal cancer and abnormal LLNs. A recent international multicenter cohort study conducted at 6 centers from the Netherlands, the United States, and Australia showed beneficial oncological outcomes when a PLND is performed along with TME surgery after NCRT in Western patients with pretreatment abnormal LLNs in terms of lower lateral local recurrence rate and local recurrence rate $[137,140]$. Patients with low rectal cancers with abnormal LLNs ( $>5 \mathrm{~mm}$ short axis in the obturator, internal iliac, external iliac, and/or common iliac basin) who underwent NCRT and TME were compared to similarly staged patients who underwent a PLND in addition to NCRT and 
TME. Between groups, the local recurrence rate was 3\% for the PLND group vs. $11 \%$ for no PLND. Disease-free and overall survival were similar, being PLND an independent significant factor for local recurrences [136].

Besides technical difficulty and potential complications, the improvement in disease-free survival rates after PLND suggests that lateral nodal disease is rather a local than a distant issue. Several Japanese centers have begun performing selective PLND in patients with enlarged ( $>7 \mathrm{~mm}$ ) LLNs after NCRT $[148,149]$. Eastern surgeons are adopting NCRT with indicated LLNDs to prevent overtreatment $[148,149]$, and Western surgeons gradually recognize that LLR is a significant issue in certain patients [10].

Another question is if intensifying RT to suspicious metastatic PLND during NCRT could replace PLND; Hartvigson et al. [150] reported that RT dose escalation to nonresected suspicious PLND during NCRT was well tolerated. Enrolled patients in this study received an RT boost to a cumulative dose of > 50.4 Gy to clinically suspicious LPND metastases based on abnormal LN size (short axis of $>0.5 \mathrm{~cm}$ ). Of 12 patients enrolled, 25\% of patients received an integrated boost to $60.2 \mathrm{~Gy}$ in 28 fractions, and 2 patients received an intraoperative radiotherapy boost of 10 Gy to either the anticipated close margins or unresectable LN areas. All patients received RT boosts of $>50.4$ Gy to 60.2 Gy in 28 fractions with good tolerance to NCRT and associated with a high rate of the short-term local control in 12 months without increasing risk of intraoperative complications.

\section{Expert comments}

According to the JSCCR surgical treatment guideline, the incidence of LPLN metastasis was reported as 30\% in patients with cT3 rectal cancer below the peritoneal reflection [24]. In my surgical practice, preoperative chemoradiotherapy used to be recommended to occasionally enlarged pelvic sidewall LN was found in cT3 distal and middle rectal cancer. As reported, more distal rectal tumors tend to have more LN metastases and more lateral lymphatic spread [151]. In National Comprehensive Cancer Network guidelines for rectal cancer [152], pelvic LN metastases have been regarded as extra-regional LN metastases, the same as paraaortic LN metastases, representing a stage IV extension of disease and does not recommend the extension of nodal dissection beyond the field of TME unless thee nodes are clinically suspicious. Although JCOG0212 trial reported that prophylactic PLND significantly reduced recurrence rate compared with TME alone (7.4\% vs. $12.6 \%$ ) without a significant increase in severe postoperative complications [146]. With the current evidence, LPNDs are interpreted as a local extension of the disease by Korean and Japanese surgeons, and TME+PLND can improve oncologic outcomes and prevent local recurrence, given that the TME approach is designed to radically remove only lymphatic regions of tumors located above the level of the levator muscles [153]. For locally advanced middle and low rectal cancer, prospectively controlled trials comparing NCRT and TME alone vs. NCRT+TME with or without PLND are needed to clarify the patient's selection criteria and indications of PLND.

The management of LPND metastases in mid and lower rectal cancer remains debated. Still, in the west, current studies tried to show RT boost can control LPND metastases in some patients and avoid technically demanding procedures to improve disease control. Which one will be less toxic; RT or PLND? RT boosts effects on the risk of immediate and delayed postoperative complications remains unclear. Although there was a low risk of acute toxicity and perioperative complications and a high local control rate in 12 months, RT boost protocol warrants further studies in patients with clinically involved nonresected LPNL [150].

Personally, after NCRT, the remaining enlarged pelvic LN should be removed, and the complete sterilization of the PLND area can be confirmed with short-term adjuvant chemotherapy. PLND remains a technically demanding procedure that risks incomplete dissection and residual tumor. We also need to develop systematic training programs with proctoring on minimally invasive approaches to perform this procedure in obese patients or a narrow pelvis and by experienced hands, given that serious complications for vascular injury during PLND. In my experience, during robotic PLND, an unforced or unintended robotic instrument may damage the pelvic artery and vein. In this situation, sometimes, we need help from a vascular surgeon and convert to open surgery.

In addition, to prevent remnant metastatic pelvic LNs, a fluorescence-guided navigation approach has been suggested [154], but from a practical point of view, its application seems to be problematic in all cases.

\section{Inguinal lymph node dissection}

The primary lymphatic drainage of the rectum follows the mesorectal, lateral pelvic, and ILNs (Fig. 21). In general, lower rectal cancer with anal canal involvement has been postulated to metastasize to the ILNs $[63,155]$. Shiratori et al. [155] suggested that DL involvement and ILN of $>8 \mathrm{~mm}$ predicted the development of ILN metastases in patients with low-lying rectal cancer. The frequency of synchronous ILN metastases to $4.5 \%-6.2 \%$ in lower rectal cancer, and the 5-year cumulative incidence rate of ILN metastases after rectal surgery was $3.4 \%$ to $4.0 \%$ in lower rectal cancer invading the anal canal $[156,157]$.

Taylor et al. [72] did not recommend a routine RT at the inguinal area. The overall rate of ILN failure in patients with lower rectal cancer extending to the anal canal treated using NCRT without elective inguinal irradiation is low. However, the morbidity of elective inguinal irradiation is significant. Still, according to Shiratori et al. [155], inguinal irradiation without ILN may be advised in patients with high suspicious ILN metastases, given that inguinal radiation seems more effective than surgical resection in controlling inguinal micrometastases. If salvage surgery is needed, ILN resection can also be performed after inguinal irradiation, but some concerns about radiation-related morbidities at the in- 


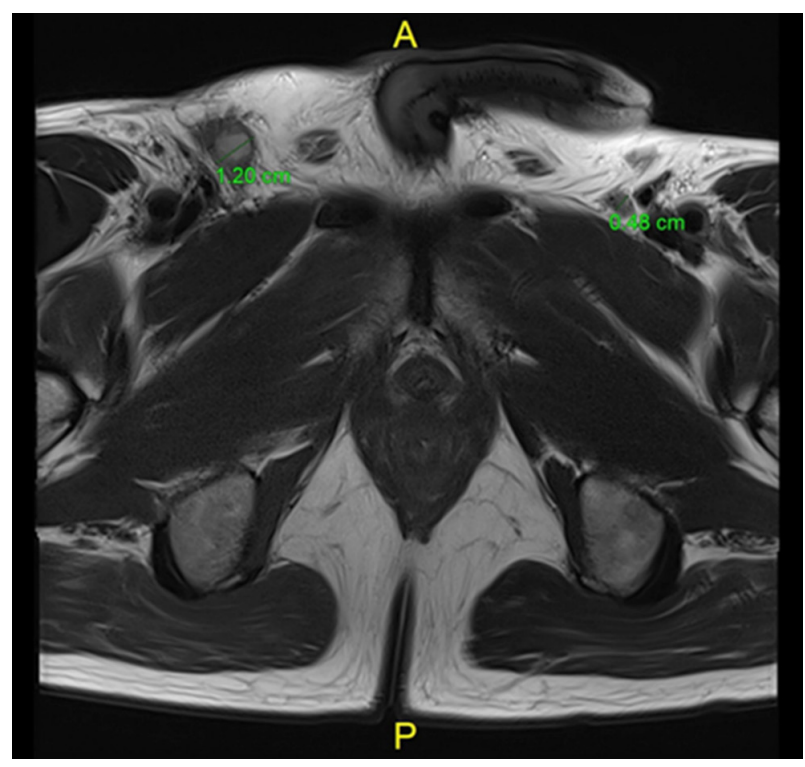

Fig. 21. Pelvic magnetic resonance imaging shows enlarged right inguinal lymph node.

guinal area are still present.

Salvage ILN dissection is also performed if ILN recurrence during the follow-up period. Yeo et al. [157] analyzed 1,226 rectal cancer patients retrospectively managed using NCRT or postNCRT in ILN metastases clinically negative mean follow-up period 66 months out of them, ILN metastases found in 7 patients with anal canal involvement. They conclude that a low ILN recurrence rate at rectal cancer patients with anal canal involvement is feasible for salvage treatment of isolated ILN recurrence and conclude that routine elective inguinal radiation is not necessary.

Based on these results, the effect and complication of ILN radiation to the inguinal area in distal rectal cancer should be carefully evaluated, and the risk of ILN metastasis recurrence should be treated promptly with salvage surgery if diagnosed.

Although routine inguinal area radiation treatment at distal rectal cancer will be unnecessary, ILN NCRT can impact tumor and control of inguinal micrometastasis for clinically and radiologically highly suspicious metastases.

The critical message for colorectal surgeons is that distal rectal cancer with anal canal involvement has a risk of metastasis to the ILN, and we must be aware of it when planning treatment strategies.

\section{Watch-and-wait \\ Introduction}

The evolution of NCRT modalities has improved clinical outcomes by downstaging and downsizing rectal cancer tumors to a complete clinical response (cCR) in $15 \%$ to $30 \%$ of patients [158]. The pioneering study by Habr-Gama et al. [159] in 2004 included 71 patients, with 5-year overall survival ranging from $85.9 \%$ to $100 \%$ in subsequent studies $[83,160,161]$.

\section{Patient selection}

Even though the gold standard treatment of rectal cancer is surgical TME, a comparative study evaluated the outcomes of patients who underwent surgery after CCR to NCRT vs. patients who enrolled in the watch-and-wait protocol, founding similar rates of non-regrowth recurrence and rectal cancer-specific survival compared with surgery [162]. Comparing surgery vs. watch-and-wait, 3-year overall survival rates were $100 \%$ vs. $88 \%(P=0.03)$, rectal cancer-specific survival rates were $100 \%$ vs. $95 \%(\mathrm{P}=0.16)$, and freedom from non-regrowth recurrence rates were $92 \%$ vs. $85 \%$ $(\mathrm{P}=0.36)$. This study revealed better outcomes for stage I/II patients undergoing watch-and-wait than those with stage III disease. Patients with more advanced stage disease were more likely to receive surgical treatment with systemic chemotherapy than the watch-and-wait group.

These results might be affected by individual inclusion criteria after CCR evaluation and the surgeon's own consideration of the risk of local regrowth disease progression vs. the potential benefit of rectal preservation and avoidance of the morbidity and mortality of surgery. A Korean comparative study by Oh et al. [163] confirmed the oncologic feasibility of local excision in ypT0-1 rectal cancer after NCRT compared to a radical resection, with no significant differences in overall recurrence-free survival or preoperative $\mathrm{T}$ stage, with favorable prognosis in rectum-sparing strategies in patients with favorable response to NCRT.

Jimenez-Rodrigues et al. [164] analyzed 88 patients with stage II and III rectal cancer who received an induction-type total neoadjuvant therapy (TNT) consisting of 8 cycles of FOLFOX or XELOX before chemoradiotherapy between 2012 and 2019. They reported that TNT using induction chemotherapy followed by chemoradiotherapy could achieve a cCR in $36.2 \%$ of patients, and watch-and-wait protocol could be advised. In this regard, approximately $1 / 3$ of patients with stage II and stage III rectal cancer can benefit from a watch-and-wait approach to preserve the rectum.

\section{Follow-up}

Nonsurgical management in a patient with $\mathrm{CCR}$ includes a close follow-up with endoscopy, digital rectal examination, and MRI but accurate strategies for tumor response determination after NCRT remain inconclusive. Patient selection to the watch-andwait protocol or SPS needs accurate prediction of ypT status by clinical assessment, endoscopy, and MRI. Although neither endoscopy nor MRI had proven adequate diagnostic performances, the combination modality shows significantly higher diagnostic performance in sensitivity (56.92\%) and accuracy (67.27\%) compared with MRI alone [6].

The Korean Society of Abdominal Radiology recently published an evidence-based guide for the MRI interpretation of complete tumor response after NCRT therapy for rectal cancer, concluding the criteria for complete tumor response on T2 may include normalization of the wall; regular, thin, hypointense scar in the luminal side with normal-appearance or homogeneous intermediate 
signal in the underlying wall; and hypointense thickening of the wall in the former tumor location and the criteria for complete tumor response on diffusion-weighted imaging (DWI) should be the absence of a hyperintense signal on high b-value DWI in the former tumor location [8].

Integrating endoscopy in clinical restaging strategy tumor regrowth enables early detection of local regrowth. In the first large multicenter registry-based study on international watch-and-wait strategies for patients with rectal cancer, local regrowth (213 of 880 patients) was most frequently diagnosed in the first 2 years of follow-up (88\%) located in the bowel wall in $97 \%$ of these patients [83]. Salvage TME was performed for $78 \%$ of local recurrences achieving $\mathrm{R} 0$ resections in $88 \%$ of cases. This publication had a 5 -year overall survival of $84.7 \%$ and 5 -year disease-specific survival of $93.8 \%$. The risk of local unsalvageable disease was small with a 5 -year disease-specific survival was $84 \%$ and 5-year overall survival of $75.4 \%$ for patients diagnosed with local regrowth, making salvage surgery an appropriate option for watch-and-wait patients with local regrowth with good oncological outcomes.

Patients from expert centers had a local regrowth rate between $10 \%$ and $30 \%$ during watch-and-wait, and the vast majority was easily amenable to salvage resection. Only very few patients suffered from a locally uncontrolled situation and metastatic disease. The 4 available series of watch-and-wait suggest that the excess risk of cancer-related death is in the order of $<2 \%$ to $3 \%$ [ 160 , 165-167].

Early tumor regrowth in patients with cCR managed entirely by watch-and-wait protocol was evaluated by Habr-Gama et al. [168]. According to their findings in 67 patients with cCR, cT2 patients developed similar initial cCR rates compared with cT3/ T4 (72\% vs. 63\%; P = 0.403). Nevertheless, early tumor regrowths were more frequent among patients with initial cT3/4 stage when compared with cT2 patients $(30 \%$ vs. $3 \%, \mathrm{P}=0.007)$. The cT2 patients who develop cCR after extended chemoradiation managed nonoperatively are less likely to develop early tumor regrowths when compared with cT3/4 patients. The cT3/4 patients should undergo more intensive follow-up after a cCR to detect early regrowths.

Perez et al. [169] also reviewed the oncological outcomes of salvage resection for local recurrences in 53 patients managed by chemoradiotherapy followed by TEM for rectal preservation. Patients with "near" complete response to chemoradiotherapy $(\leq 3$ $\mathrm{cm}$; ycT1-2N0) were offered TEM, and salvage surgery was attempted in the event of a local recurrence. All patients $(n=12)$ who developed local recurrence presented initial unfavorable pathological features (ypT2 or ypT3, poor differentiation [tumor grade], lymphovascular invasion, or perineural invasion) in the TEM specimen. Eight patients underwent APR as salvage surgery with CRM+ in 7 of 8 (87\%); 4 of these patients developed local rerecurrence during follow-up. The 2-year local recurrence-free survival was $77 \%$, and a 2 -year local rerecurrence-free survival of $60 \%$. The high rates of incomplete TME after local recurrence
(R1) and the high local rerecurrence rate after salvage resection suggests considering the completion of TME in patients with unfavorable pathological features after near-complete response treated by TEM [167].

A single-center study reviewed local recurrence retrospectively in 78 rectal cancer patients under watch-and-wait surveillance. Twenty-three patients developed local recurrence and underwent salvage surgery, while 55 remain under watch-and-wait surveillance. Compared to 46 patients (37\%) who underwent TME after NCRT for persistent disease, the local recurrence group had lower rectal tumors $(4.5 \pm 3 \mathrm{~cm}$ vs. $2.3 \pm 2 \mathrm{~cm})$, although anastomotic leaks, perioperative and oncological outcomes rates were similar between groups [170].

Recent systematic reviews and meta-analyses have concluded that although the local recurrence rate of the watch-and-wait group could be higher than in the TME group when promptly treated by salvage therapy, the watch-and-wait strategy could achieve similar oncologic outcomes as the TME approach. Salvage surgery (APR, LAR, TaTME) is possible in $83.8 \%$ of patients who develop a tumor regrowth with the "watch-and-wait" approach with no difference in overall survival and disease-free survival between patients who received immediate surgery without differences in relative risk of overall mortality and relative risk of disease-specific mortality $[122,171,172]$.

\section{Expert comments}

As colorectal surgeons, we have experienced patients who show a cCR after NCRT. Furthermore, the adoption of induction or consolidation chemotherapy in total neoadjuvant treatments [164] has increased the incidence of cCR. The watch-and-wait strategy also got more attention because salvage surgery for regrowing tumors during follow-up did not compromise an oncologic outcome.

Although we consider that the evaluation criteria of cCR have not been standardized, the watch-and-wait strategy can be carefully recommended in highly selected patients. Salvage TME could be restricted for patients developing local recurrence to reduce unnecessary proctectomies in a significant proportion of patients with initial cCR. As we can expect, this treatment plan will get more attractive as a treatment option for surgeons and patients in the future.

\section{Prevention of anastomotic leakage}

Innovations in minimally invasive surgery and the variety of anastomotic stapler devices have lowered the rate of AL in the last few years. Despite advances in surgical practice, the rates of $\mathrm{AL}$ have remained around $10 \%$ to $15 \%$. A retrospective study of 103 patients was conducted in a single center in Japan to identify risk factors for AL; the univariate analysis showed a younger age, advanced stage, deeper depth of tumor invasion, larger tumor circumference, longer operation time, and early postoperative diarrhea were associated with AL [173]. The etiology of AL is multifactorial, but one of the most crucial risk factors is under the sur- 
geon's control, whose abilities remain the most critical resource to avoid complications. Some technical tips and tricks are worth being reminded of for better outcomes:

(1) Mobilization of splenic flexure and high tie of the inferior mesentery artery could be required in some patients if the patient lacks a redundant sigmoid colon to achieve a tension-free anastomosis.

(2) The distal transection of the rectum should be done with a single stapling firing if possible. A systematic review and metaanalysis suggest that 2 firings imply a higher rate of anastomotic leak than a single firing after laparoscopic rectal surgery with a double stapling technique [54]. Anastomotic leak occurred in $3.5 \%$ of the cases (17 of 491) where 1 stapler firing was used vs. $6.7 \%$ of the cases (50 of 786) in which 2 firings were needed.

(3) Placement of a TAT decreases the rate of clinical AL (3\%-7\% vs. $12 \%-13 \%$ in patients without it) in patients with TAT and a lower risk of reoperation related to leakage (risk ratio, 0.19; $95 \%$ CI, 0.08-0.46) when the TAT was placed [174-176].

(4) Indocyanine green fluorescence angiography (ICG FA) is now being used to evaluate blood supply at the anastomotic site. Intraoperative use of ICG FA is associated with a lower incidence of AL after LAR. The benefit of ICG FA may be that it could identify patients with high risk for AL [177]. The ongoing MRC/ NIHR IntAct study will focus on evaluating the use of ICG FA in rectal cancer surgery and also explore the role of the microbiome in $\mathrm{AL}$ and the predictive value of preoperative $\mathrm{CT}$ angiography/ perfusion scanning [177].

(5) Stapled rings like doughnuts should be inspected for anastomotic completeness, and an air leak test with intraoperative colonoscopy must confirm the integrity of the anastomosis.

(6) DST can be reinforced according to the surgeon's criteria. Transanal or transabdominal reinforcement of colorectal anastomosis by additional sutures reduces the incidence of postoperative complications associated with AL in high-risk patients [67].

(7) A diverting ileostomy was performed in all patients undergoing ULAR and some undergoing LAR to divert the fecal stream away from the immature anastomosis [10].

For patients with a high risk of AL who refuse to have a protective ileostomy, an alternative CAA can be performed following the Turnbull and Cutait pull-through or delayed CAA [32, 178]. In this technique, after rectal resection for SPS, the left colon completely mobilized to be tension-free exteriorized a few centimeters below the anal verge and fixed to perianal skin by 2 stitches and kept in place through the anal canal by the resting pressure of the anal canal (Fig. 22). The exteriorized segment is visualized daily and covered in gauze. On postoperative days 6 to 10, the exteriorized colon is resected. A delayed hand-sewn CAA is performed at the anal margin level, preserving the adhesions created between the colonic serosa pelvic tissues the anal canal wall contribute to reducing anastomotic leak. This technique reduces the need for diverting ileostomy without increasing postoperative morbidity rates and avoiding the need for permanent stomas in the short term compared with standard CAA with protective ileostomy followed by ileostomy closure [32]. In a recent systematic review of 8 observational studies ( 409 patients), with no AL in 6 out of 8 studies, the mortality rate was $\leq 3 \%$, poor fecal continence was reported in $<30 \%$ of patients, and the need for permanent stoma was $\leq 2 \%$ [179]. Although this technique is popular in Europe and Latin America, it is still not popular in Korea and Japan.

To improve knowledge in AL prevention, the ongoing IMARI trial [180] will evaluate 3 prophylactic interventions (mechanical bowel preparation with oral antibiotics, tailored total splenic flexure mobilization, and intraoperative ICG FA) combined with a standardized pathway for early detection and active management of AL in a multicenter prospective clinical effectiveness trial. The multi-interventional program will focus on anastomotic integrity confirmed by CT-scan at 1 year postoperatively, protocol compliance and association with AL, temporary and permanent stoma rate, reintervention rate, QoL, and functional outcome.

\section{Expert comments}

Safe anastomosis and prevention of early and delayed AL tension-
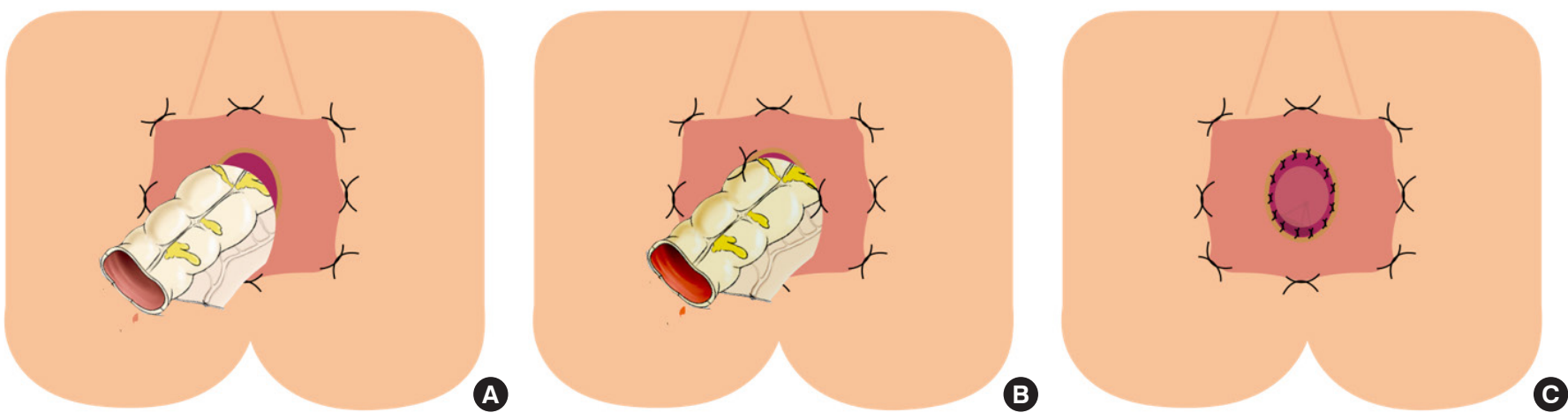

Fig. 22. Two-stage Turnbull-Cutait pull-through coloanal anastomosis. (A) Exteriorization of the colon. (B) Colon fixation to the anal margin. (C) Hand-sewn coloanal anastomosis. Modified from Biondo et al. [32] with permission from JAMA. 
free and good blood supply are mandatory in the proximal colon. During IMV high ligation below the inferior border of the pancreas, the arc of Riolan or the meandering artery should be identified and preserved. Its inadvertent ligation near the IMV origin can compromise the blood supply to the splenic flexure of the colon, leading to mucosal congestion, ischemia, colitis, and necrosis with a high risk of AL reoperation [181, 182].

\section{CONCLUSION}

Colorectal surgeons constantly confront issues regarding oncological safety and functional outcomes when facing distal rectal cancer. The extent of the disease should be thoroughly assessed with modern imaging methods to determine a tailored treatment plan. Furthermore, the patient's QoL must be considered, making their participation in the decision-making process is crucial, as this makes them aware of the potential impact of the surgical treatment on their bowel, urinary, and sexual function.

The distal mesorectum and covering fascia should be separated sharply from the surrounding structure, such as urogenital organs, pelvic plexus, and neurovascular bundles from the underlying pelvic floor. In this subject, we suggest to young colorectal surgeons a couple of tips with my previous publications regarding the step-by-step approach for rectal mobilization [1], the gate approach to the pelvic floor [74], and customized Denonvilliers' excision; technical tips introduced to help to perform a complete TME in distal rectal cancer. Hopefully, these didactically developed techniques will contribute to a good understanding of the anatomy of the anorectum and pelvic autonomic nerve structures and improve the oncologic outcomes and high QoL in patients with distal rectal cancer.

An essential tool for a colorectal surgeon is the knowledge of anorectal anatomy and function to perform a sharp anatomical dissection based on surgical anatomy for a guaranteed oncological and functional, safe deep pelvic dissection. In addition to that, new technological innovation keeps developing, and we should adopt these techniques to improve our patient outcomes. It is important to remember that all new techniques imply a learning curve, so updated proctoring education programs are mandatory to keep along with innovations. We firmly believe every colorectal surgeon could be a master surgeon, and we encourage them to keep learning the surgical treatment of rectal cancer.

\section{CONFLICT OF INTEREST}

No potential conflict of interest relevant to this article was reported.

\section{REFERENCES}

1. Kim NK, Kim HS, Alessa M, Torky R. Optimal complete rectum mobilization focused on the anatomy of the pelvic fascia and au- tonomic nerves: 30 years of experience at Severance Hospital. Yonsei Med J 2021;62:187-99.

2. Church JM, Raudkivi PJ, Hill GL. The surgical anatomy of the rectum: a review with particular relevance to the hazards of rectal mobilisation. Int J Colorectal Dis 1987;2:158-66.

3. Kim NK. Anatomic basis of sharp pelvic dissection for curative resection of rectal cancer. Yonsei Med J 2005;46:737-49.

4. Bogach J, Tsai S, Zbuk K, Wong R, Grubac V, Coates A, et al. Quality of preoperative pelvic computed tomography (CT) and magnetic resonance imaging (MRI) for rectal cancer in a region in Ontario: a retrospective population-based study. J Surg Oncol 2018;117:1038-42.

5. Zhou Y, Shao W, Lu W. Diagnostic value of endorectal ultrasonography for rectal carcinoma: a meta-analysis. J Cancer Res Ther 2014;10 Suppl:319-22.

6. Cho MS, Kim H, Han YD, Hur H, Min BS, Baik SH, et al. Endoscopy and magnetic resonance imaging-based prediction of ypT stage in patients with rectal cancer who received chemoradiotherapy: results from a prospective study of 110 patients. Medicine (Baltimore) 2019;98:e16614.

7. Al-Sukhni E, Milot L, Fruitman M, Beyene J, Victor JC, Schmocker S, et al. Diagnostic accuracy of MRI for assessment of T category, lymph node metastases, and circumferential resection margin involvement in patients with rectal cancer: a systematic review and meta-analysis. Ann Surg Oncol 2012;19:2212-23.

8. Park SH, Cho SH, Choi SH, Jang JK, Kim MJ, Kim SH, et al. MRI assessment of complete response to preoperative chemoradiation therapy for rectal cancer: 2020 guide for practice from the Korean Society of Abdominal Radiology. Korean J Radiol 2020;21: 812-28.

9. Kim CH, Yeom SS, Kwak HD, Lee SY, Ju JK, Kim YJ, et al. Clinical outcomes of patients with locally advanced rectal cancer with persistent circumferential resection margin invasion after preoperative chemoradiotherapy. Ann Coloproctol 2019;35:72-82.

10. Kang SB, Park JW, Jeong SY, Nam BH, Choi HS, Kim DW, et al. Open versus laparoscopic surgery for mid or low rectal cancer after neoadjuvant chemoradiotherapy (COREAN trial): shortterm outcomes of an open-label randomised controlled trial. Lancet Oncol 2010;11:637-45.

11. Kim JY, Kim YW, Kim NK, Hur H, Lee K, Min BS, et al. Pelvic anatomy as a factor in laparoscopic rectal surgery: a prospective study. Surg Laparosc Endosc Percutan Tech 2011;21:334-9.

12. Kyo K, Sameshima S, Takahashi M, Furugori T, Sawada T. Impact of autonomic nerve preservation and lateral node dissection on male urogenital function after total mesorectal excision for lower rectal cancer. World J Surg 2006;30:1014-9.

13. Nakagoe T, Ishikawa H, Sawai T, Tsuji T, Tanaka K, Hidaka S, et al. Survival and recurrence after a sphincter-saving resection and abdominoperineal resection for adenocarcinoma of the rectum at or below the peritoneal reflection: a multivariate analysis. Surg Today 2004;34:32-9.

14. Nash GM, Weiss A, Dasgupta R, Gonen M, Guillem JG, Wong 
WD. Close distal margin and rectal cancer recurrence after sphincter-preserving rectal resection. Dis Colon Rectum 2010; 53:1365-73.

15. Zeng WG, Liu MJ, Zhou ZX, Wang ZJ. A distal resection margin of $\leq 1 \mathrm{~mm}$ and rectal cancer recurrence after sphincter-preserving surgery: the role of a positive distal margin in rectal cancer surgery. Dis Colon Rectum 2017;60:1175-83.

16. Stelzner S, Holm T. Extralevator abdominoperineal excision. Coloproctology 2020;42;37-46.

17. Holm T, Ljung A, Häggmark T, Jurell G, Lagergren J. Extended abdominoperineal resection with gluteus maximus flap reconstruction of the pelvic floor for rectal cancer. Br J Surg 2007;94: 232-8.

18. Miles WE. A method of performing abdomino-perineal excision for carcinoma of the rectum and of the terminal portion of the pelvic colon. Lancet 1908;172:1812-3.

19. Jayne D, Pigazzi A, Marshall H, Croft J, Corrigan N, Copeland J, et al. Effect of robotic-assisted vs conventional laparoscopic surgery on risk of conversion to open laparotomy among patients undergoing resection for rectal cancer: the ROLARR randomized clinical trial. JAMA 2017;318:1569-80.

20. Kim TH, Jeong SY, Choi DH, Kim DY, Jung KH, Moon SH, et al. Lateral lymph node metastasis is a major cause of locoregional recurrence in rectal cancer treated with preoperative chemoradiotherapy and curative resection. Ann Surg Oncol 2008;15:72937.

21. Beets GL, Figueiredo NL, Habr-Gama A, van de Velde CJ. A new paradigm for rectal cancer: organ preservation. Introducing the International Watch \& Wait Database (IWWD). Eur J Surg Oncol 2015;41:1562-4.

22. Choy KT, Yang T, Prabhakaran S, Heriot A, Kong JC, Warrier SK. Comparing functional outcomes between transanal total mesorectal excision (TaTME) and laparoscopic total mesorectal excision (LaTME) for rectal cancer: a systematic review and meta-analysis. Int J Colorectal Dis 2021;36:1163-74.

23. Heald RJ. A new approach to rectal cancer. Br J Hosp Med 1979; 22:277-81.

24. Hashiguchi Y, Muro K, Saito Y, Ito Y, Ajioka Y, Hamaguchi T, et al. Japanese Society for Cancer of the Colon and Rectum (JSCCR) guidelines 2019 for the treatment of colorectal cancer. Int J Clin Oncol 2020;25:1-42.

25. Lowry AC, Simmang CL, Boulos P, Farmer KC, Finan PJ, Hyman $\mathrm{N}$, et al. Consensus statement of definitions for anorectal physiology and rectal cancer: report of the Tripartite Consensus Conference on Definitions for Anorectal Physiology and Rectal Cancer, Washington, D.C., May 1, 1999. Dis Colon Rectum 2001; 44:915-9.

26. Wibe A, Rendedal PR, Svensson E, Norstein J, Eide TJ, Myrvold $\mathrm{HE}$, et al. Prognostic significance of the circumferential resection margin following total mesorectal excision for rectal cancer. $\mathrm{Br} \mathrm{J}$ Surg 2002;89:327-34.

27. Adam IJ, Mohamdee MO, Martin IG, Scott N, Finan PJ, John- ston $\mathrm{D}$, et al. Role of circumferential margin involvement in the local recurrence of rectal cancer. Lancet 1994;344:707-11.

28. Rullier E, Laurent C, Bretagnol F, Rullier A, Vendrely V, Zerbib F. Sphincter-saving resection for all rectal carcinomas: the end of the 2-cm distal rule. Ann Surg 2005;241:465-9.

29. Lindsey I, Guy RJ, Warren BF, Mortensen NJ. Anatomy of Denonvilliers' fascia and pelvic nerves, impotence, and implications for the colorectal surgeon. Br J Surg 2000;87:1288-99.

30. Kong M, Chen H, Xin Y, Jiang Y, Han Y, Sheng H. High ligation of the inferior mesenteric artery and anastomotic leakage in anterior resection for rectal cancer: a systematic review and metaanalysis of randomized controlled trial studies. Colorectal Dis 2021;23:614-24.

31. Hajibandeh S, Hajibandeh S, Maw A. Meta-analysis and trial sequential analysis of randomized controlled trials comparing high and low ligation of the inferior mesenteric artery in rectal cancer surgery. Dis Colon Rectum 2020;63:988-99.

32. Biondo S, Trenti L, Espin E, Bianco F, Barrios O, Falato A, et al. Two-stage Turnbull-Cutait pull-through coloanal anastomosis for low rectal cancer: a randomized clinical trial. JAMA Surg 2020;155:e201625.

33. Lyttle JA, Parks AG. Intersphincteric excision of the rectum. Br J Surg 1977;64:413-6.

34. Yang SY, Kim NK. Robotic partial excision of levator-ani muscle for locally advanced low rectal cancer invading ipsilateral pelvic floor. Ann Coloproctol 2020;36:415-6.

35. Fleshman JW, Wexner SD, Anvari M, LaTulippe JF, Birnbaum EH, Kodner IJ, et al. Laparoscopic vs. open abdominoperineal resection for cancer. Dis Colon Rectum 1999;42:930-9.

36. Wang HS, Lin JK, Yang SH, Jiang JK, Chen WS, Lin TC. Prospective study of the functional results of transanal endoscopic microsurgery. Hepatogastroenterology 2003;50:1376-80.

37. Neary P, Makin GB, White TJ, White E, Hartley J, MacDonald A, et al. Transanal endoscopic microsurgery: a viable operative alternative in selected patients with rectal lesions. Ann Surg Oncol 2003;10:1106-11.

38. Gavagan JA, Whiteford MH, Swanstrom LL. Full-thickness intraperitoneal excision by transanal endoscopic microsurgery does not increase short-term complications. Am J Surg 2004;187:6304.

39. Lee W, Lee D, Choi S, Chun H. Transanal endoscopic microsurgery and radical surgery for T1 and T2 rectal cancer. Surg Endosc 2003;17:1283-7.

40. Hwang Y, Yoon YS, Bong JW, Choi HY, Song IH, Lee JL, et al. Long-term transanal excision outcomes in patients with $\mathrm{T} 1 \mathrm{rec}-$ tal cancer: comparative analysis of radical resection. Ann Coloproctol 2019;35:194-201.

41. Jayne DG, Guillou PJ, Thorpe H, Quirke P, Copeland J, Smith AM, et al. Randomized trial of laparoscopic-assisted resection of colorectal carcinoma: 3-year results of the UK MRC CLASICC Trial Group. J Clin Oncol 2007;25:3061-8.

42. van der Pas MH, Haglind E, Cuesta MA, Fürst A, Lacy AM, Hop 
WC, et al. Laparoscopic versus open surgery for rectal cancer (COLOR II): short-term outcomes of a randomised, phase 3 trial. Lancet Oncol 2013;14:210-8.

43. Fleshman J, Branda ME, Sargent DJ, Boller AM, George VV, Abbas MA, et al. Disease-free survival and local recurrence for laparoscopic resection compared with open resection of stage II to III rectal cancer: follow-up results of the ACOSOG Z6051 randomized controlled trial. Ann Surg 2019;269:589-95.

44. Stevenson A, Solomon MJ, Brown C, Lumley JW, Hewett P, Clouston AD, et al. Disease-free survival and local recurrence after laparoscopic-assisted resection or open resection for rectal cancer: the Australasian Laparoscopic Cancer of the Rectum randomized clinical trial. Ann Surg 2019;269:596-602.

45. Park JW, Kang SB, Hao J, Lim SB, Choi HS, Kim DW, et al. Open versus laparoscopic surgery for mid or low rectal cancer after neoadjuvant chemoradiotherapy (COREAN trial): 10-year follow-up of an open-label, non-inferiority, randomised controlled trial. Lancet Gastroenterol Hepatol 2021;6:569-77.

46. Kim JC, Lee JL, Alotaibi AM, Yoon YS, Kim CW, Park IJ. Robotassisted intersphincteric resection facilitates an efficient sphincter-saving in patients with low rectal cancer. Int J Colorectal Dis 2017;32:1137-45.

47. Nacion A, Park YY, Yang SY, Kim NK. Critical and challenging issues in the surgical management of low-lying rectal cancer. Yonsei Med J 2018;59:703-16.

48. Grass JK, Chen CC, Melling N, Lingala B, Kemper M, Scognamiglio $\mathrm{P}$, et al. Robotic rectal resection preserves anorectal function: Systematic review and meta-analysis. Int J Med Robot 2021;17:e2329.

49. Roodbeen SX, Penna M, Mackenzie H, Kusters M, Slater A, Jones OM, et al. Transanal total mesorectal excision (TaTME) versus laparoscopic TME for MRI-defined low rectal cancer: a propensity score-matched analysis of oncological outcomes. Surg Endosc 2019;33:2459-67.

50. Zuhdy M, Elmore U, Shams N, Hegazy M, Roshdy S, Eldamshety $\mathrm{O}$, et al. Transanal versus laparoscopic total mesorectal excision: a comparative prospective clinical trial from two centers. J Laparoendosc Adv Surg Tech A 2020;30:769-76.

51. Lee JM, Han YD, Cho MS, Hur H, Min BS, Lee KY, et al. Prediction of transabdominal total mesorectal excision difficulty according to the angle of pelvic floor muscle. Surg Endosc 2020;34: 3043-50.

52. Yasui M, Takemasa I, Miyake Y, Hata T, Ikeda M, Miyake Y, et al. Tumor Size as an independent risk factor for postoperative complications in laparoscopic low anterior resection for advanced rectal cancer: a multicenter Japanese study. Surg Laparosc Endosc Percutan Tech 2017;27:98-103.

53. He J, Yao HB, Wang CJ, Yang QY, Qiu JM, Chen JM, et al. Metaanalysis of laparoscopic anterior resection with natural orifice specimen extraction (NOSE-LAR) versus abdominal incision specimen extraction (AISE-LAR) for sigmoid or rectal tumors. World J Surg Oncol 2020;18:215.
54. Balciscueta Z, Uribe N, Caubet L, López M, Torrijo I, Tabet J, et al. Impact of the number of stapler firings on anastomotic leakage in laparoscopic rectal surgery: a systematic review and metaanalysis. Tech Coloproctol 2020;24:919-25.

55. Lacy AM, Tasende MM, Delgado S, Fernandez-Hevia M, Jimenez M, De Lacy B, et al. Transanal total mesorectal excision for rectal cancer: outcomes after 140 patients. J Am Coll Surg 2015;221: 415-23.

56. Heald RJ. A new solution to some old problems: transanal TME. Tech Coloproctol 2013;17:257-8.

57. Motson RW, Whiteford MH, Hompes R, Albert M, Miles WF; Expert Group. Current status of trans-anal total mesorectal excision (TaTME) following the Second International Consensus Conference. Colorectal Dis 2016;18:13-8.

58. Penna M, Hompes R, Arnold S, Wynn G, Austin R, Warusavitarne J, et al. Incidence and risk factors for anastomotic failure in 1594 patients treated by transanal total mesorectal excision: results from the international TaTME Registry. Ann Surg 2019;269: 700-11.

59. Sparreboom CL, Komen N, Rizopoulos D, van Westreenen HL, Doornebosch PG, Dekker J, et al. Transanal total mesorectal excision: how are we doing so far? Colorectal Dis 2019;21:767-74.

60. Sylla P, Knol JJ, D’Andrea AP, Perez RO, Atallah SB, Penna M, et al. Urethral injury and other urologic injuries during transanal total mesorectal excision: an international collaborative study. Ann Surg 2021;274:e115-25.

61. Dayal S, Moran B. LOREC: the English Low Rectal Cancer National Development Programme. Br J Hosp Med (Lond) 2013;74: 377-80.

62. Persiani R, Agnes A, Belia F, D'Ugo D, Biondi A. The learning curve of TaTME for mid-low rectal cancer: a comprehensive analysis from a five-year institutional experience. Surg Endosc 2021;35:6190-200.

63. Larsen SG, Pfeffer F, Kørner H; Norwegian Colorectal Cancer Group. Norwegian moratorium on transanal total mesorectal excision. Br J Surg 2019;106:1120-1.

64. Wasmuth HH, Faerden AE, Myklebust TÅ, Pfeffer F, Norderval S, Riis R, et al. Transanal total mesorectal excision for rectal cancer has been suspended in Norway. Br J Surg 2020;107:121-30.

65. Deijen CL, Velthuis S, Tsai A, Mavroveli S, de Lange-de Klerk ES, Sietses C, et al. COLOR III: a multicentre randomised clinical trial comparing transanal TME versus laparoscopic TME for mid and low rectal cancer. Surg Endosc 2016;30:3210-5.

66. van der Heijden J, van de Pas K, van den Broek F, van Dielen F, Slooter GD, Maaskant-Braat A. Oncological and functional outcomes of transanal total mesorectal excision in a teaching hospital in the Netherlands. Ann Coloproctol 2021 Jun 29 [Epub]. https://doi.org/10.3393/ac.2020.00773.0110.

67. Balkarov AA, Alekseev MV, Rybakov EG, Frolov SA, Achkasov SI. Profilaktika nesostoyatel'nosti kolorektal'nogo anastomoza putem ego ukrepleniya (rezultaty randomizirovannogo issledovaniya) [Prevention of colorectal anastomotic leakage using its 
reinforcement (results of the randomized study)]. Khirurgiia (Mosk) 2021;(7):18-23.

68. Alimova I, Chernyshov S, Nagudov M, Rybakov E. Comparison of oncological and functional outcomes and quality of life after transanal or laparoscopic total mesorectal excision for rectal cancer: a systematic review and meta-analysis. Tech Coloproctol 2021;25:901-13.

69. Kusters M, Marijnen CA, van de Velde CJ, Rutten HJ, Lahaye MJ, Kim JH, et al. Patterns of local recurrence in rectal cancer: a study of the Dutch TME trial. Eur J Surg Oncol 2010;36:470-6.

70. Park IJ, Kim JC. Adequate length of the distal resection margin in rectal cancer: from the oncological point of view. J Gastrointest Surg 2010;14:1331-7.

71. Kanso F, Lefevre JH, Svrcek M, Chafai N, Parc Y, Tiret E. Partial mesorectal excision for rectal adenocarcinoma: morbidity and oncological outcome. Clin Colorectal Cancer 2016;15:82-90.

72. Taylor FG, Quirke P, Heald RJ, Moran B, Blomqvist L, Swift I, et al. Preoperative high- resolution magnetic resonance imaging can identify good prognosis stage I, II, and III rectal cancer best managed by surgery alone: a prospective, multicenter, European study. Ann Surg 2011;253:711-9.

73. Kennedy ED, Simunovic M, Jhaveri K, Kirsch R, Brierley J, Drolet $\mathrm{S}$, et al. Safety and feasibility of using magnetic resonance imaging criteria to identify patients with "good prognosis" rectal cancer eligible for primary surgery: the phase 2 nonrandomized QuickSilver clinical trial. JAMA Oncol 2019;5:961-6.

74. Sauri F, Cho MS, Kim NK. Gate approach at deep anterolateral pelvic dissection. Surg Oncol 2021;37:101535.

75. Teramoto T, Watanabe M, Kitajima M. Per anum intersphincteric rectal dissection with direct coloanal anastomosis for lower rectal cancer: the ultimate sphincter-preserving operation. Dis Colon Rectum 1997;40(10 Suppl):S43-7.

76. Watanabe M, Teramoto T, Hasegawa H, Kitajima M. Laparoscopic ultralow anterior resection combined with per anum intersphincteric rectal dissection for lower rectal cancer. Dis Colon Rectum 2000;43(10 Suppl):S94-7.

77. Law WL, Chu KW. Anterior resection for rectal cancer with mesorectal excision: a prospective evaluation of 622 patients. Ann Surg 2004;240:260-8.

78. Park EJ, Baik SH, Kang J, Hur H, Min BS, Lee KY, et al. The impact of postoperative complications on long-term oncologic outcomes after laparoscopic low anterior resection for rectal cancer. Medicine (Baltimore) 2016;95:e3271.

79. Nakagoe T, Ishikawa H, Sawai T, Tsuji T, Takeshita H, Nanashima A, et al. Oncological outcome of ultra-low anterior resection with total mesorectal excision for carcinoma of the lower third of the rectum: comparison of intrapelvic double-stapled anastomosis and transanal coloanal anastomosis. Hepatogastroenterology 2005;52:1692-7.

80. Lee JB, Kim HS, Ham A, Chang JS, Shin SJ, Beom SH, et al. Role of preoperative chemoradiotherapy in clinical stage II/III rectal cancer patients undergoing total mesorectal excision: a retro- spective propensity score analysis. Front Oncol 2021;10:609313.

81. Kim HG, Kim HS, Yang SY, Han YD, Cho MS, Hur H, et al. Early recurrence after neoadjuvant chemoradiation therapy for locally advanced rectal cancer: characteristics and risk factors. Asian J Surg 2021;44:298-302.

82. Ikoma N, You YN, Bednarski BK, Rodriguez-Bigas MA, Eng C, Das $P$, et al. Impact of recurrence and salvage surgery on survival after multidisciplinary treatment of rectal cancer. J Clin Oncol 2017;35:2631-8.

83. van der Valk M, Hilling DE, Bastiaannet E, Meershoek-Klein Kranenbarg E, Beets GL, Figueiredo NL, et al. Long-term outcomes of clinical complete responders after neoadjuvant treatment for rectal cancer in the International Watch \& Wait Database (IWWD): an international multicentre registry study. Lancet 2018;391:2537-45.

84. Sauri F, Sakr A, Kim HS, Alessa M, Torky R, Zakarneh E, et al. Does the timing of protective ileostomy closure post-low anterior resection have an impact on the outcome? A retrospective study. Asian J Surg 2021;44:374-9.

85. Gavaruzzi T, Pace U, Giandomenico F, Pucciarelli S, Bianco F, Selvaggi F, et al. Colonic J-pouch or straight colorectal reconstruction after low anterior resection for rectal cancer: impact on quality of life and bowel function. A multicenter prospective randomized study. Dis Colon Rectum 2020;63:1511-23.

86. Baker JW. Low end to side rectosigmoidal anastomosis; description of technic. Arch Surg 1950;61:143-57.

87. Ziv Y, Zbar A, Bar-Shavit Y, Igov I. Low anterior resection syndrome (LARS): cause and effect and reconstructive considerations. Tech Coloproctol 2013;17:151-62.

88. Nakada I, Kawasaki S, Sonoda Y, Watanabe Y, Tabuchi T. Abdominal stapled side-to-end anastomosis (Baker type) in low and high anterior resection: experiences and results in 69 consecutive patients at a regional general hospital in Japan. Colorectal Dis 2004;6:165-70.

89. Hou S, Wang Q, Zhao S, Liu F, Guo P, Ye Y. Safety and efficacy of side-to-end anastomosis versus colonic J-pouch anastomosis in sphincter-preserving resections: an updated meta-analysis of randomized controlled trials. World J Surg Oncol 2021;19:130.

90. Elghamrini Y, Ibrahim Hassan M, Sabry Abdel Samee K, Aly Khalil A. Critical surgical errors by junior fellows and trainees in low rectal cancer surgery: how to overcome?(a cross-sectional study). Ann Med Surg (Lond) 2021;62:440-5.

91. Sakr A, Yang SY, Kang JH, Cho MS, Han YD, Min BS, et al. Oncologic safety and bowel function after ultralow anterior resection with or without intersphincteric resection for low lying rectal cancer: comparative cross sectional study. J Surg Oncol 2019 Dec 3 [Epub]. https://doi.org/10.1002/jso.25791.

92. Zhuang CL, Zhang FM, Wang Z, Jiang X, Wang F, Liu ZC. Precision functional sphincter-preserving surgery (PPS) for ultralow rectal cancer: a natural orifice specimen extraction (NOSE) surgery technique. Surg Endosc 2021;35:476-85.

93. Zhuang CL, Liu Z, Zhang FM, Wang Z, Liu Q, Liu ZC. Surgical 
key points of precision functional sphincter-preserving surgery. Zhonghua Wei Chang Wai Ke Za Zhi 2020;23:597-600.

94. Shang J, Leibrandt K, Giataganas P, Vitiello V, Seneci CA, Wisanuvej P, et al. A single-port robotic system for transanal microsurgery-design and validation. IEEE Robot Autom Lett. 2017;2: 1510-7.

95. Schiessel R, Karner-Hanusch J, Herbst F, Teleky B, Wunderlich M. Intersphincteric resection for low rectal tumours. Br J Surg 1994; 81:1376-8.

96. Yamamoto S, Ito M, Okuda J, Fujii S, Yamaguchi S, Yoshimura K, et al. Laparoscopic surgery for stage $0 / \mathrm{I}$ rectal carcinoma: shortterm outcomes of a single-arm phase II trial. Ann Surg 2013;258: 283-8.

97. Yamada K, Saiki Y, Takano S, Iwamoto K, Tanaka M, Fukunaga $\mathrm{M}$, et al. Long-term results of intersphincteric resection for low rectal cancer in Japan. Surg Today 2019;49:275-85.

98. Fujimoto Y, Akiyoshi T, Kuroyanagi H, Konishi T, Ueno M, Oya $\mathrm{M}$, et al. Safety and feasibility of laparoscopic intersphincteric resection for very low rectal cancer. J Gastrointest Surg 2010;14: 645-50.

99. Kim NK, Kim MS, Al-Asari SF. Update and debate issues in surgical treatment of middle and low rectal cancer. J Korean Soc Coloproctol 2012;28:230-40.

100. Akagi Y, Kinugasa T, Shirouzu K. Intersphincteric resection for very low rectal cancer: a systematic review. Surg Today 2013;43: 838-47.

101. Rullier E, Sa Cunha A, Couderc P, Rullier A, Gontier R, Saric J. Laparoscopic intersphincteric resection with coloplasty and coloanal anastomosis for mid and low rectal cancer. Br J Surg 2003; 90:445-51.

102. Kim NK, Kim YW, Cho MS. Intersphincteric resection and coloanal reconstruction. In: Chang G, editor. Rectal cancer: modern approaches to treatment. Singapore: Springer Cham; 2018. p. 191-211.

103. Piozzi GN, Park H, Kim JS, Choi HB, Lee TH, Baek SJ, et al. Anatomic landmarks for transabdominal robotic-assisted intersphincteric dissection for ultralow anterior resection. Dis Colon Rectum 2021;64:e87-8.

104. Zhang B, Zhuo GZ, Zhao K, Zhao Y, Gao DW, Zhu J, et al. Cumulative incidence and risk factors of permanent stoma after intersphincteric resection for ultralow rectal cancer. Dis Colon Rectum 2022;65:66-75.

105. Narihiro S, Miura N, Nishizawa Y, Hasegawa H, Ikeda K, Teramura K, et al. Delorme surgery for colonic mucosal prolapse after intersphincteric resection. Surg Today 2021;51:916-22.

106. Alessa M, Bae HW, Alawfi H, Sakr A, Sauri F, Kim NK. Neorectal mucosal prolapse after intersphincteric resection for low-lying rectal cancer: a case report. Ann Coloproctol 2021;37(Suppl 1):S15-7.

107. Chau A, Frasson M, Debove C, Maggiori L, Panis Y. Colonic prolapse after intersphincteric resection for very low rectal cancer: a report of 12 cases. Tech Coloproctol 2016;20:701-5.
108. Guraieb-Trueba M, Helber AR, Marks JH. Full-thickness neorectal prolapse after transanal transabdominal proctosigmoidectomy for low rectal cancer: a cohort study. Colorectal Dis 2018; 20:593-6.

109. Denost Q, Moreau JB, Vendrely V, Celerier B, Rullier A, Assenat $\mathrm{V}$, et al. Intersphincteric resection for low rectal cancer: the risk is functional rather than oncological. A 25-year experience from Bordeaux. Colorectal Dis 2020;22:1603-13.

110. Park JS, Kim NK, Kim SH, Lee KY, Lee KY, Shin JY, et al. Multicentre study of robotic intersphincteric resection for low rectal cancer. Br J Surg 2015;102:1567-73.

111. Park IJ, Kim JC. Intersphincteric resection for patients with lowlying rectal cancer: oncological and functional outcomes. Ann Coloproctol 2018;34:167-74.

112. Kim NK, Sugihara K, Liang JT. Surgical treatment of colorectal cancer: Asian perspectives on optimization and standardization. Singapore: Springer Singapore; 2018.

113. Tsukada Y, Ito M, Watanabe K, Yamaguchi K, Kojima M, Hayashi R, et al. Topographic anatomy of the anal sphincter complex and levator ani muscle as it relates to intersphincteric resection for very low rectal disease. Dis Colon Rectum 2016;59:426-33.

114. Lee JM, Kim NK. Essential anatomy of the anorectum for colorectal surgeons focused on the gross anatomy and histologic findings. Ann Coloproctol 2018;34:59-71.

115. Yang SY, Cho MS, Kim NK. Outcomes of robotic partial excision of the levator ani muscle for locally advanced low rectal cancer invading the ipsilateral pelvic floor at the anorectal ring level. Int J Med Robot 2021;17:e2310.

116. AlAsari SF, Lim D, Kim NK. Hemi-levator excision to provide greater sphincter preservation in low rectal cancer. Int J Colorectal Dis 2013;28:1727-8.

117. Noh GT, Han J, Cheong C, Han YD, Kim NK. Novel anal sphincter saving procedure with partial excision of levator-ani muscle in rectal cancer invading ipsilateral pelvic floor. Ann Surg Treat Res 2017;93:195-202.

118. Shihab OC, Heald RJ, Holm T, How PD, Brown G, Quirke P, et al. A pictorial description of extralevator abdominoperineal excision for low rectal cancer. Colorectal Dis 2012;14:e655-60.

119. Campos FG, Habr-Gama A, Nahas SC, Perez RO. Abdominoperineal excision: evolution of a centenary operation. Dis Colon Rectum 2012;55:844-53.

120. de Campos-Lobato LF, Stocchi L, Dietz DW, Lavery IC, Fazio VW, Kalady MF. Prone or lithotomy positioning during an abdominoperineal resection for rectal cancer results in comparable oncologic outcomes. Dis Colon Rectum 2011;54:939-46.

121. Park S, Hur H, Min BS, Kim NK. Short-term outcomes of an extralevator abdominoperineal resection in the prone position compared with a conventional abdominoperineal resection for advanced low rectal cancer: the early experience at a single institution. Ann Coloproctol 2016;32:12-9.

122. Tao Y, Han JG, Wang ZJ. Extralevator abdominoperineal excision for advanced low rectal cancer: where to go. World J Gas- 
troenterol 2020;26:3012-23.

123. Mesquita-Neto J, Mouzaihem H, Macedo F, Heilbrun LK, Weaver DW, Kim S. Perioperative and oncological outcomes of abdominoperineal resection in the prone position vs the classic lithotomy position: a systematic review with meta-analysis. J Surg Oncol 2019;119:979-86.

124. Sabbagh C, Fumery M, Mauvais F, Regimbeau JM. The prone position for performing perineal dissection during extralevator abdominoperineal resection: a necessary waste of time? Dis Colon Rectum 2016;59:353-6.

125. Tao Y, Han JG, Wang ZJ. Comparison of perineal morbidity between biologic mesh reconstruction and primary closure following extralevator abdominoperineal excision: a systematic review and meta-analysis. Int J Colorectal Dis 2021;36:893-902.

126. Zaheer Ahmad N, Abbas MH, Al-Naimi N, Parvaiz A. Metaanalysis of biological mesh reconstruction versus primary perineal closure after abdominoperineal excision of rectal cancer. Int J Colorectal Dis 2021;36:477-92.

127. Meyer J, Roos E, Abbassi Z, Toso C, Ris F, Buchs NC. The role of perineal application of prophylactic negative-pressure wound therapy for prevention of wound-related complications after abdomino-perineal resection: a systematic review. Int J Colorectal Dis 2021;36:19-26.

128. Qi XY, Cui M, Liu MX, Xu K, Tan F, Yao ZD, et al. Extralevator abdominoperineal excision versus abdominoperineal excision for low rectal cancer: a meta-analysis. Chin Med J (Engl) 2019; 132:2446-56.

129. Zhou X, Sun T, Xie H, Zhang Y, Zeng H, Fu W. Extralevator abdominoperineal excision for low rectal cancer: a systematic review and meta-analysis of the short-term outcome. Colorectal Dis 2015;17:474-81.

130. Habr-Gama A, São Julião GP, Mattacheo A, de Campos-Lobato LF, Aleman E, Vailati BB, et al. Extralevator abdominal perineal excision versus standard abdominal perineal excision: impact on quality of the resected specimen and postoperative morbidity. World J Surg 2017;41:2160-7.

131. Asplund D, Prytz M, Bock D, Haglind E, Angenete E. Persistent perineal morbidity is common following abdominoperineal excision for rectal cancer. Int J Colorectal Dis 2015;30:1563-70.

132. Aggarwal N, Seshadri RA, Arvind A, Jayanand SB. Perineal wound complications following extralevator abdominoperineal excision: experience of a regional cancer center. Indian J Surg Oncol 2018;9:211-4.

133. West NP, Anderin C, Smith KJ, Holm T, Quirke P; European Extralevator Abdominoperineal Excision Study Group. Multicentre experience with extralevator abdominoperineal excision for low rectal cancer. Br J Surg 2010;97:588-99.

134. Kasai S, Kagawa H, Shiomi A, Hino H, Manabe S, Yamaoka Y, et al. Advantages of robotic abdominoperineal resection compared with laparoscopic surgery: a single-center retrospective study. Surg Today 2021 Aug 21 [Epub]. https://doi.org/10.1007/s00595021-02359-6.
135. Matsuda T, Yamashita K, Hasegawa H, Takiguchi G, Urakawa N, Yamamoto $\mathrm{M}$, et al. Transperineal minimally invasive abdominoperineal resection for low rectal cancer: standardized technique and clinical outcomes. Surg Endosc. 2021;35:7236-45.

136. Emile SH, Elfeki H, Shalaby M, Sakr A, Kim NK. Outcome of lateral pelvic lymph node dissection with total mesorectal excision in treatment of rectal cancer: a systematic review and metaanalysis. Surgery 2021;169:1005-15.

137. Kroon HM, Malakorn S, Dudi-Venkata NN, Bedrikovetski S, Liu J, Kenyon-Smith T, et al. Local recurrences in western low rectal cancer patients treated with or without lateral lymph node dissection after neoadjuvant (chemo)radiotherapy: an international multi-centre comparative study. Eur J Surg Oncol 2021;47:24419.

138. Ogura A, Konishi T, Cunningham C, Garcia-Aguilar J, Iversen H, Toda S, et al. Neoadjuvant (chemo)radiotherapy with total mesorectal excision only is not sufficient to prevent lateral local recurrence in enlarged nodes: results of the multicenter lateral node study of patients with low cT3/4 rectal cancer. J Clin Oncol 2019;37:33-43.

139. Ogura A, Konishi T, Beets GL, Cunningham C, Garcia-Aguilar J, Iversen $\mathrm{H}$, et al. Lateral nodal features on restaging magnetic resonance imaging associated with lateral local recurrence in low rectal cancer after neoadjuvant chemoradiotherapy or radiotherapy. JAMA Surg 2019;154:e192172.

140. Cribb B, Kong J, McCormick J, Warrier S, Heriot A. Meta-analysis of direct-to-surgery lateral pelvic lymph node dissection for rectal cancer. Colorectal Dis 2021;23:1687-98.

141. Fujita S, Yamamoto S, Akasu T, Moriya Y. Lateral pelvic lymph node dissection for advanced lower rectal cancer. Br J Surg 2003; 90:1580-5.

142. Wang X, Qiu A, Liu X, Shi Y. Total mesorectal excision plus lateral lymph node dissection vs TME on rectal cancer patients: a meta-analysis. Int J Colorectal Dis 2020;35:997-1006.

143. Ito M, Kobayashi A, Fujita S, Mizusawa J, Kanemitsu Y, Kinugasa Y, et al. Urinary dysfunction after rectal cancer surgery: Results from a randomized trial comparing mesorectal excision with and without lateral lymph node dissection for clinical stage II or III lower rectal cancer (Japan Clinical Oncology Group Study, JCOG0212). Eur J Surg Oncol 2018;44:463-8.

144. Sasaki T, Ito Y, Ohue M, Kanemitsu Y, Kobatake T, Ito M, et al. Postoperative chemoradiotherapy after local resection for highrisk T1 to T2 low rectal cancer: results of a single-arm, multi-institutional, phase II clinical trial. Dis Colon Rectum 2017;60:91421.

145. Fujita S, Akasu T, Mizusawa J, Saito N, Kinugasa Y, Kanemitsu Y, et al. Postoperative morbidity and mortality after mesorectal excision with and without lateral lymph node dissection for clinical stage II or stage III lower rectal cancer (JCOG0212): results from a multicentre, randomised controlled, non-inferiority trial. Lancet Oncol 2012;13:616-21.

146. Fujita S, Mizusawa J, Kanemitsu Y, Ito M, Kinugasa Y, Komori K, 
et al. Mesorectal excision with or without lateral lymph node dissection for clinical stage II/III lower rectal cancer (JCOG0212): a multicenter, randomized controlled, noninferiority trial. Ann Surg 2017;266:201-7.

147. Kusters M, van de Velde CJ, Beets-Tan RG, Akasu T, Fujita S, Yamamoto $S$, et al. Patterns of local recurrence in rectal cancer: a single-center experience. Ann Surg Oncol 2009;16:289-96.

148. Akiyoshi T, Ueno M, Matsueda K, Konishi T, Fujimoto Y, Nagayama S, et al. Selective lateral pelvic lymph node dissection in patients with advanced low rectal cancer treated with preoperative chemoradiotherapy based on pretreatment imaging. Ann Surg Oncol 2014;21:189-96.

149. Matsuda T, Sumi Y, Yamashita K, Hasegawa H, Yamamoto M, Matsuda Y, et al. Outcomes and prognostic factors of selective lateral pelvic lymph node dissection with preoperative chemoradiotherapy for locally advanced rectal cancer. Int J Colorectal Dis 2018;33:367-74.

150. Hartvigson PE, Apisarnthanarax S, Schaub S, Cohen S, Bernier G, Koh WJ, et al. Radiation therapy dose escalation to clinically involved pelvic sidewall lymph nodes in locally advanced rectal cancer. Adv Radiat Oncol 2019;4:478-86.

151. Steup WH, Moriya Y, van de Velde CJ. Patterns of lymphatic spread in rectal cancer. A topographical analysis on lymph node metastases. Eur J Cancer 2002;38:911-8.

152. NCCN Guidelines Version 2.2021 Panel Members, Rectal Cancer. NCCN Clinical Practice Guidelines in Oncology (NCCN Guidelines $^{\circledR}$ ) [Internet]. Plymouth Meeting: National Comprehensive Cancer Network; 2021 [cited 2021 Oct 15]. Available from: https://www.nccn.org/professionals/physician_gls/pdf/ rectal.pdf.

153. Bae S, Kim NK. Is the never-ending story still unsolved? Beyond the long debate about lateral pelvic lymph node dissection in rectal cancer. Dis Colon Rectum 2021;64:253-5.

154. Kim HJ, Park JS, Choi GS, Park SY, Lee HJ. Fluorescence-guided robotic total mesorectal excision with lateral pelvic lymph node dissection in locally advanced rectal cancer: a video presentation. Dis Colon Rectum 2017;60:1332-3.

155. Shiratori H, Nozawa H, Kawai K, Hata K, Tanaka T, Kaneko M, et al. Risk factors and therapeutic significance of inguinal lymph node metastasis in advanced lower rectal cancer. Int J Colorectal Dis 2020;35:655-64.

156. Taylor N, Crane C, Skibber J, Feig B, Ellis L, Vauthey JN, et al. Elective groin irradiation is not indicated for patients with adenocarcinoma of the rectum extending to the anal canal. Int J Radiat Oncol Biol Phys 2001;51:741-7.

157. Yeo SG, Lim HW, Kim DY, Kim TH, Kim SY, Baek JY, et al. Is elective inguinal radiotherapy necessary for locally advanced rectal adenocarcinoma invading anal canal? Radiat Oncol 2014; 9:296.

158. Habr-Gama A, Perez RO, Wynn G, Marks J, Kessler H, GamaRodrigues J. Complete clinical response after neoadjuvant chemoradiation therapy for distal rectal cancer: characterization of clinical and endoscopic findings for standardization. Dis Colon Rectum 2010;53:1692-8.

159. Habr-Gama A, Perez RO, Nadalin W, Sabbaga J, Ribeiro U Jr, Silva e Sousa AH Jr, et al. Operative versus nonoperative treatment for stage 0 distal rectal cancer following chemoradiation therapy: long-term results. Ann Surg 2004;240:711-7.

160. Habr-Gama A, Gama-Rodrigues J, São Julião GP, Proscurshim I, Sabbagh C, Lynn PB, et al. Local recurrence after complete clinical response and watch and wait in rectal cancer after neoadjuvant chemoradiation: impact of salvage therapy on local disease control. Int J Radiat Oncol Biol Phys 2014;88:822-8.

161. Hupkens B, Martens MH, Stoot JH, Berbee M, Melenhorst J, Beets-Tan RG, et al. Quality of life in rectal cancer patients after chemoradiation: watch-and-wait policy versus standard resection. A matched-controlled study. Dis Colon Rectum 2017;60: 1032-40.

162. Beard BW, Rettig RL, Ryoo JJ, Parker RA, McLemore EC, Attaluri V. Watch-and-Wait compared to operation for patients with complete response to neoadjuvant therapy for rectal cancer. J Am Coll Surg 2020;231:681-92.

163. Oh SY, Park IJ, Kim YI, Lee JL, Kim CW, Yoon YS, et al. Comparison between local excision and radical resection for the treatment of rectal cancer in ypT0-1 patients: an analysis of the clinicopathological factors and survival rates. Cancers (Basel) 2021;13: 4823.

164. Jimenez-Rodriguez RM, Quezada-Diaz F, Hameed I, Kalabin A, Patil S, Smith JJ, et al. Organ preservation in patients with rectal cancer treated with total neoadjuvant therapy. Dis Colon Rectum 2021;64:1463-70.

165. Appelt AL, Pløen J, Harling H, Jensen FS, Jensen LH, Jørgensen JC, et al. High-dose chemoradiotherapy and watchful waiting for distal rectal cancer: a prospective observational study. Lancet Oncol 2015;16:919-27.

166. Maas M, Beets-Tan RG, Lambregts DM, Lammering G, Nelemans PJ, Engelen SM, et al. Wait-and-see policy for clinical complete responders after chemoradiation for rectal cancer. J Clin Oncol 2011;29:4633-40.

167. Smith JD, Ruby JA, Goodman KA, Saltz LB, Guillem JG, Weiser $\mathrm{MR}$, et al. Nonoperative management of rectal cancer with complete clinical response after neoadjuvant therapy. Ann Surg 2012; 256:965-72.

168. Habr-Gama A, São Julião GP, Gama-Rodrigues J, Vailati BB, Ortega C, Fernandez LM, et al. Baseline T classification predicts early tumor regrowth after nonoperative management in distal rectal cancer after extended neoadjuvant chemoradiation and initial complete clinical response. Dis Colon Rectum 2017;60: 586-94.

169. Perez RO, Habr-Gama A, São Julião GP, Proscurshim I, Fernandez LM, de Azevedo RU, et al. Transanal endoscopic microsurgery (TEM) following neoadjuvant chemoradiation for rectal cancer: outcomes of salvage resection for local recurrence. Ann Surg Oncol 2016;23:1143-8. 
170. Nasir I, Fernandez L, Vieira P, Parés O, Santiago I, Castillo-Martin $\mathrm{M}$, et al. Salvage surgery for local regrowths in Watch \& Wait: are we harming our patients by deferring the surgery? Eur J Surg Oncol 2019;45:1559-66.

171. Kong JC, Guerra GR, Warrier SK, Ramsay RG, Heriot AG. Outcome and salvage surgery following "watch and wait" for rectal cancer after neoadjuvant therapy: a systematic review. Dis Colon Rectum 2017;60:335-45.

172. On J, Shim J, Aly EH. Systematic review and meta-analysis on outcomes of salvage therapy in patients with tumour recurrence during 'watch and wait' in rectal cancer. Ann R Coll Surg Engl 2019;101:441-52.

173. Hiraki M, Tanaka T, Ikeda O, Sadashima E, Kimura N, Nakamura $S$, et al. Retrospective risk analysis for anastomotic leakage following laparoscopic rectal cancer surgery in a single institute. J Gastrointest Cancer 2020;51:908-13.

174. Wang S, Zhang Z, Liu M, Li S, Jiang C. Efficacy of transanal tube placement after anterior resection for rectal cancer: a systematic review and meta-analysis. World J Surg Oncol 2016;14:92.

175. Dumble C, Morgan T, Wells CI, Bissett I, O'Grady G. The impact of transanal tube design for preventing anastomotic leak in anterior resection: a systematic review and meta-analysis. Tech Coloproctol 2021;25:59-68.

176. Choy KT, Yang T, Heriot A, Warrier SK, Kong JC. Does rectal tube/transanal stent placement after an anterior resection for rectal cancer reduce anastomotic leak? A systematic review and meta-analysis. Int J Colorectal Dis 2021;36:1123-32.

177. Armstrong G, Croft J, Corrigan N, Brown JM, Goh V, Quirke P, et al. IntAct: intra-operative fluorescence angiography to prevent anastomotic leak in rectal cancer surgery. A randomized controlled trial. Colorectal Dis 2018;20:O226-34.

178. Jarry J, Faucheron JL, Moreno W, Bellera CA, Evrard S. Delayed colo-anal anastomosis is an alternative to prophylactic diverting stoma after total mesorectal excision for middle and low rectal carcinomas. Eur J Surg Oncol 2011;37:127-33.

179. Portale G, Popesc GO, Parotto M, Cavallin F. Delayed colo-anal anastomosis for rectal cancer: pelvic morbidity, functional results and oncological outcomes. A systematic review. World J Surg 2019;43:1360-9.

180. Slooter MD, Talboom K, Sharabiany S, van Helsdingen C, van Dieren S, Ponsioen CY, et al. IMARI: multi-Interventional program for prevention and early Management of Anastomotic leakage after low anterior resection in Rectal cancer patIents. Rationale and study protocol. BMC Surg 2020;20:240.

181. Al-Asari SF, Lim D, Min BS, Kim NK. The relation between inferior mesenteric vein ligation and collateral vessels to splenic flexure: anatomical landmarks, technical precautions and clinical significance. Yonsei Med J 2013;54:1484-90.

182. Toh J, Matthews R, Kim SH. Arc of Riolan-preserving splenic flexure takedown during anterior resection: potentially critical to prevent acute anastomotic ischemia. Dis Colon Rectum 2018; 61:411-4. 